Collection SFN 11 (2010) 153-176

(C) Owned by the authors, published by EDP Sciences, 2010

DOI: $10.1051 / \mathrm{sfn} / 201011010$

\title{
Structure des solutions de polyélectrolytes : apport de la diffusion des rayons $X$ et des neutrons aux petits angles
}

\author{
J. Combet
}

Institut Charles Sadron, Université de Strasbourg, CNRS UPR 22, 23 rue du Loess, BP. 84047, 67034 Strasbourg Cedex, France

\begin{abstract}
Résumé. Dans cette contribution, nous présentons l'apport de la diffusion des rayons $\mathrm{X}$ et des neutrons à l'étude de la structure des solutions de polyélectrolytes. Le but n'est pas d'être exhaustif sur les dernières avancées réalisées dans ce domaine, mais plutôt de comprendre les principales caractéristiques observées sur les courbes de diffusion ainsi que les renseignements que l'on peut en tirer. Nous verrons que suivant les densités de longueur de diffusion des différents constituants, il est possible d'extraire des informations sur l'état de dispersion ainsi que sur la conformation moyenne des macroions en solution. Nous nous limiterons toutefois à l'étude des systèmes flexibles, hydrophiles, en régime semidilué, sans sel ajouté.
\end{abstract}

\section{INTRODUCTION}

Les polyélectrolytes (PEs) sont des composés macromoléculaires qui possèdent des groupements ionisables. Après dissociation dans un solvant polaire comme de l'eau, ils présentent des charges de même signe, distribuées le long du polyion. À chaque groupement ionisé correspond un contreion de charge opposée qui se déplace plus ou moins librement dans la solution. Les répulsions électrostatiques entre monomères le long d'une même chaine conduisent à une conformation moyenne étirée, tandis que les interactions interchaînes permettent une stabilisation de la solution. Ces propriétés, qui diffèrent de celles rencontrées dans les polymères neutres, sont à l'origine d'un certain nombre d'applications. La structure de ces solutions est un point crucial qu'il est nécessaire de comprendre pour maîtriser les propriétés de ces composés. Elle concerne la conformation moyenne des polyions, leur état de dispersion, mais aussi la distribution des contreions en solution. De ce point de vue, la diffusion de rayonnement aux petits angles joue un rôle extrêmement important car elle permet de sonder des échelles spatiales parfaitement adaptées.

Dans cette contribution, nous allons essayer de comprendre les courbes de diffusion mesurées, et voir les informations structurales que l'on peut en tirer. Nous présenterons dans un premier temps les différentes classes de PEs (partie 2) et introduirons les paramètres caractéristiques rencontrés dans ces solutions ainsi que les grandeurs physiques importantes (partie 3).

Nous décrirons ensuite les principes de base de la diffusion aux petits angles dans les systèmes à plusieurs constituants, en soulignant plus particulièrement, le rôle central joué par la variation de contraste. Nous introduirons les fonctions de structure partielles qui contiennent toute l'information sur la structure des différents constituants. Nous présenterons les méthodes utilisées pour extraire le facteur de forme des polyions, ainsi que les fonctions de corrélations intermoléculaires (partie 4).

Nous présenterons alors des mesures expérimentales réalisées sans condition de contraste particulière, ainsi que les mesures des fonctions de structure partielles associées aux polyions et aux contreions. Nous nous intéresserons plus particulièrement à l'effet de la concentration et du taux de charge sur les courbes observées (partie 5).

This is an Open Access article distributed under the terms of the Creative Commons Attribution-Noncommercial License 3.0, which permits unrestricted use, distribution, and reproduction in any noncommercial medium, provided the original work is properly cited. 


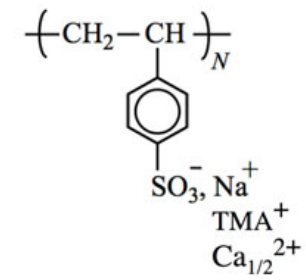

PSSNa, PSSCa ${ }_{1 / 2}$, PSSTMA<smiles>CCC(O)C(C)CCOS(=O)(=O)O[N+]#N</smiles>

NaPVS<smiles>CCC(CC(C)C)C(=O)NC(C)(C)C[Se]</smiles>

AMAMPS

Figure 1. Polystyrène sulfonate (PSSNa, $\mathrm{PSSCa}_{1 / 2}$ et PSSTMA), alcool polyvinylique partiellement estérifié (NaPVS), poly(acrylamide- co-sodium-2-acrylamido-2-methylpropane sulfonate (AMAMPS). Les contreions associés sont les ions monovalents sodium $\left(\mathrm{Na}^{+}\right)$et tétramethyle ammonium $\left(\mathrm{TMA}^{+}\right.$de formule $\left.\left(\mathrm{CH}_{3}\right)_{4} \mathrm{~N}^{+}\right)$ainsi que les ions divalents calcium $\left(\mathrm{Ca}^{2+}\right)$. Suivant l'information que l'on cherche à déterminer, les macroions, mais aussi parfois les contreions, peuvent être hydrogénés, et/ou deutériés.

Enfin, nous nous attarderons sur la mesure et l'analyse du facteur de forme des chaînes en solution, ainsi qu'à la détermination des corrélations intermoléculaires (partie 6).

\section{CLASSIFICATION DES POLYÉLECTROLYTES}

Il existe différentes façons de classer les PEs. Les plus couramment utilisées consistent à distinguer :

- le signe de la charge portée par les macroions. On parle de polycations ou de polyanions suivant que les monomères sont chargés positivement ou négativement.

- la nature chimique des groupements ionisables. Suivant leur caractère acide/base fort ou acide/base faible, on parle de PE fort ou de PE faible. Dans le premier cas, tous les groupements ionisables sont chargés. Dans le second, le taux de dissociation, ou le taux de charge chimique, dépend du pH de la solution. Pour les PEs forts, la position des charges le long de la chaîne est fixée lors de la synthèse chimique du composé (PE gelés, ou «quenched»). La distribution des charges est statique. Dans le cas des PEs faibles, la distribution des charges est dynamique (PEs recuits, ou «annealed») et fluctue avec le temps.

- le nombre de groupements chargés le long du polyions par rapport au nombre total de monomères (taux de charge chimique). On parle de PEs faiblement ou fortement chargés.

- l'hydrophobie et l'hydrophilie des monomères (ionisables ou non). Lorsque les interactions sont principalement de nature électrostatique, on parle de PEs hydrophiles. On parle de PEs hydrophobes dans le cas contraire.

D'autres classifications sont envisageables comme celles distinguant l'origine synthétique ou naturelle des macromolécules (PE naturels ou synthétiques), le côté rigide ou flexible des macroions (PE rigides, flexibles), ou encore l'architecture macromoléculaire (PE linéaires, en anneaux, branchés en forme d'étoile ou de peigne)...

Dans cette contribution, nous nous focaliserons sur des solutions aqueuses de PEs synthétiques linéaires, forts, hydrophiles et flexibles en absence de sel ajouté. Les PEs que nous considèrerons sont présentés sur la figure 1.

\section{PARAMÈTRES ET GRANDEURS PHYSIQUES CARACTÉRISTIQUES}

Parmi les paramètres importants caractérisant les chaînes de $\mathrm{PE}$, on note avant tout, la taille $b$ du monomère (de l'ordre de $2.5 \AA$ dans les systèmes vinyliques), le degré de polymérisation moyen $N$ (nombre moyen de monomères par chaîne), la distance moyenne entre charges le long de la chaîne $l$, ainsi que le taux de charge (ou fraction de charge) chimique $f$. Ce dernier est défini comme le 
rapport entre le nombre de monomères ionisés sur le nombre total de monomères, ou encore comme le rapport $b / l(0 \leq f \leq 1)$. Parmi les autres paramètres principaux, on trouve également la valence $Z$ des contreions, ainsi que la polymolécularité des chaînes. Dans le cas des PEs forts qui nous intéressent plus particulièrement, tous ces paramètres sont fixés lors de la synthèse chimique du composé. Les solutions de PEs sont caractérisées par leur concentration en monomères $c_{p}$ (mol/L).

Les macroions peuvent être considérés comme des copolymères statistiques portant des groupements ionisés (chargés), des groupements ionisables non dissociés (neutres), ainsi que des groupements non ionisables de nature chimique différente. Chacun de ces constituants peut présenter un caractère hydrophile ou hydrophobe plus ou moins marqué. La structure des solutions résulte alors de la balance entre les répulsions électrostatiques qui tendent à étirer les macromolécules, et les interactions hydrophobes qui conduisent à leur effondrement. Dans ce qui suit, nous nous limiterons à des cas relativement simples, pour lesquels le caractère hydrophobe sera négligé. Nous considèrerons que l'électrostatique domine complètement les interactions dans le système (PE hydrophile).

Les chaînes de PEs sont évidemment sensibles à la nature du solvant qui les entoure $\left(\mathrm{H}_{2} \mathrm{O}\right.$ généralement). Celui-ci doit présenter un caractère polaire suffisamment important, et une constante diélectrique $\left(\varepsilon_{r}\right)$ élevée pour permettre l'ionisation des chaînes. On définit la longueur de Bjerrum $l_{B}$, comme la distance à laquelle l'énergie électrostatique d'interaction entre deux charges élémentaires est similaire à l'énergie d'agitation thermique :

$$
l_{B}=e_{0}^{2} / 4 \pi \varepsilon_{0} \varepsilon_{r} k_{B} T .
$$

Dans l'eau pure à $25^{\circ} \mathrm{C}, \varepsilon_{r} \approx 80$ et $l_{B}=7.14 \AA$.

Lorsque la fraction de charge chimique $f$ est élevée (PE fortement chargés), le potentiel électrostatique au niveau des chaînes peut devenir important. Une partie de contreions se retrouve alors piégée dans leur voisinage proche. On parle alors de contreions libres, et de contreions condensés. Ce phénomène de condensation des contreions décrit par Manning-Oosawa [1,2], conduit à une renormalisation de la charge. En pratique, les contreions se condensent de façon à ne conserver en moyenne qu'une une charge tous les $l_{B}$ (ou $Z l_{B}$ dans le cas de contreions de valence multiple). La charge effective (ou fraction de charge effective) $f_{\text {eff }}$ portée par le polyion est égale à $b / Z l_{B}$. On définit le paramètre de charge de Manning comme $\zeta=l_{B} / l$. Pour $\zeta \leq 1 / Z$, la charge nette portée par les chaînes est égale à la charge chimique (régime de couplage faible). Lorsque $\zeta>1 / Z$, le phénomène de condensation apparaît, les contreions s'accumulent autour des chaînes de façon à ce que $\zeta$ soit maintenu à $1 / Z$ (régime de couplage fort). La charge nette est alors égale à la charge effective. Le seuil de condensation de Manning-Oosawa correspond à la frontière entre ces deux régimes. Pour $Z=1$, il se produit lorsque la distance entre charges chimiques $l$ est égale à la longueur de Bjerrum $l_{B}$. Le taux de charge correspondant est alors égal à $b / l_{B}$.

L'évolution de la charge nette en fonction de la charge chimique $f$ est schématiquement représentée sur la figure 2. Cette description n'est en théorie valable que pour des PEs rigides de symétrie cylindrique à très faible concentration. Elle peut être remise en cause dans le cas des PEs flexibles qui présentent une conformation beaucoup plus complexe. Des mesures de pression osmotique sur l'AMAMPS ont néanmoins montré que la charge effective était très proche de celle prévue par la théorie de ManningOosawa [3]. Elle constitue donc une première approximation acceptable et présente de surcroît, une extrême simplicité.

\section{PE ET DIFFUSION AUX PETITS ANGLES}

Une solution de PEs est un système ternaire composé de polyions et de contreions (soluté), et du solvant. Dans une expérience de diffusion, chacun de ces constituants contribue à l'intensité diffusée. Aux petits angles, la résolution spatiale est suffisamment faible pour que l'on puisse négliger les fluctuations de densité. La section efficace différentielle cohérente par unité de volume $\left(\Sigma^{c o h}\left(\vec{q}, c_{p}\right)\right.$ en $\left.\mathrm{cm}^{-1}\right)$ 


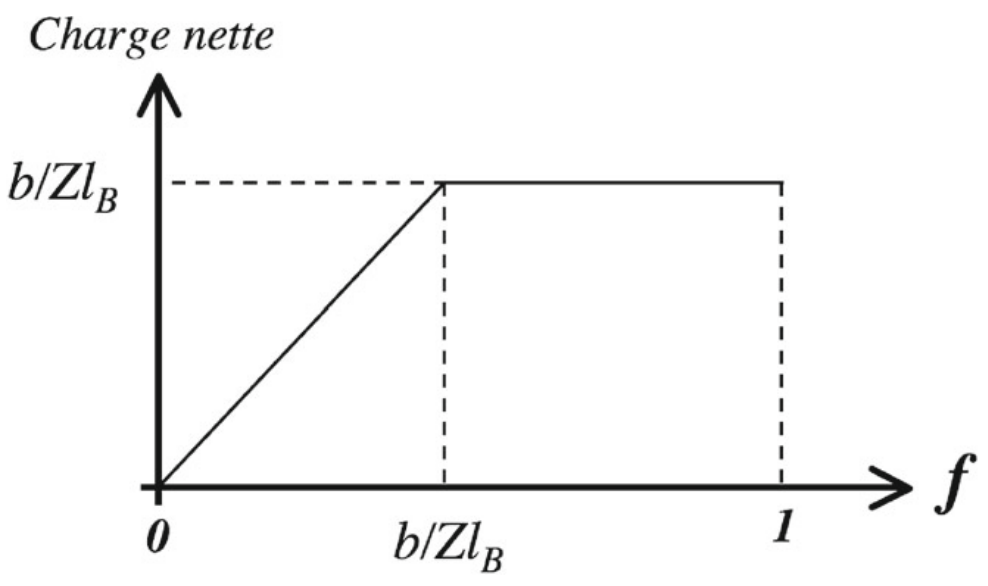

Figure 2. Evolution de la charge nette en fonction de la charge chimique $f$. Le seuil de condensation de ManningOosawa correspond à $f=b / Z l_{B}$.

qui contient toute l'information sur l'organisation structurale des diffuseurs élémentaires, s'exprime alors comme une combinaison linéaire des fonctions de structure partielles des constituants du soluté

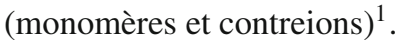

Lors d'une expérience de diffusion aux petits angles, on mesure une section efficace différentielle totale par unité de volume $\Sigma^{\text {total }}(q)$ qui s'écrit :

$$
\Sigma^{\text {total }}(q)=\Sigma^{\text {coh }}(q)+\Sigma^{B}(q)
$$

$\Sigma^{B}(q)$ est un «bruit de fond» qui doit être soustrait des données expérimentales afin d'extraire $\Sigma^{c o h}(q)$. Il comprend d'une part la diffusion du solvant (que l'on peut considérer constante sur la gamme de $q$ explorée), mais également la diffusion incohérente du soluté dans le cas de la diffusion de neutrons. La contribution du solvant peut être estimée à partir de la mesure de sa section efficace $\Sigma^{S}(q)$ et de sa fraction volumique $\Phi_{s}$. La contribution incohérente du soluté (polyions et contreions) s'écrit pour un taux de charge chimique $f=1$ :

$$
c_{p} N_{a} \sigma^{i n c} / 4 \pi
$$

$\Sigma^{B}(q)$ prend alors la forme :

$$
\Sigma^{B}(q)=\Phi_{s} \cdot \Sigma^{S}(q)+c_{p} N_{a} \sigma^{i n c} / 4 \pi .
$$

Dans ces expressions, $c_{p}$ est la concentration en monomères $\left(\mathrm{mol} . \mathrm{cm}^{-3}\right) ; N_{a}$, le nombre d'Avogadro $\left(\mathrm{mol}^{-1}\right) ; \sigma^{i n c}$, la section efficace incohérente du monomère avec son contreion $\left(\mathrm{cm}^{2}\right)$. Pour des taux de charge chimiques inférieurs $f<1$, il faut calculer séparément les contributions incohérentes des différents types de monomères. Nous ne discuterons par la suite, que de la section efficace différentielle cohérente par unité de volume $\left(\Sigma^{c o h}(q)\right)$, considérant que $\Sigma^{B}(q)$ a été correctement soustrait.

\subsection{Fonctions de structure partielles}

Le soluté ayant deux constituants (monomères et contreions), $\Sigma^{c o h}(q)$ est une combinaison linéaire des fonctions (ou facteurs) de structure partielles momomère/monomère $\left(S_{m m}\right)$, contreion/contreion $\left(S_{c c}\right)$ et

\footnotetext{
1 Par la suite, nous n'indiquerons plus systématiquement le caractère vectoriel du vecteur de diffusion. Les mesures étant isotropes, elles ne dépendent que son module. De même, et pour une question de clarté, la dépendance en concentration de $\Sigma^{c o h}\left(q, c_{p}\right)$ et de toutes les fonctions de structures associées ne sera mentionnée que si nécessaire.
} 
monomère/contreion $\left(S_{m c}\right)$ :

$$
\Sigma^{c o h}(q)=K_{m}^{2} S_{m m}(q)+K_{c}^{2} S_{c c}(q)+2 K_{m} K_{c} S_{m c}(q) .
$$

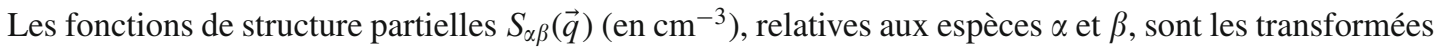
de Fourier spatiales des fonctions de corrélation des densités $n_{\alpha}$ et $n_{\beta}$ :

$$
S_{\alpha \beta}(\vec{q})=\frac{1}{V} \sum_{i, j}\left\langle\exp \left[i \vec{q}\left(\vec{r}_{i}^{\alpha}-\vec{r}_{j}^{\beta}\right)\right]\right\rangle .
$$

Dans cette expression, la sommation est à réaliser sur tous les couples monomères-monomères $\left(S_{m m}\right)$, contreions-contreions $\left(S_{c c}\right)$ et monomères-contreions $\left(S_{m c}\right)$. Ces fonctions contiennent toute l'information sur l'organisation respective $\left(S_{m m}, S_{c c}\right)$ et croisée $\left(S_{m c}\right)$ des différents constituants. $S_{m m}$ combine des termes intra et intermoléculaire relatifs aux chaînes. Il en est de même pour les contreions. Cette notion ne prend néanmoins tout son sens, que lorsqu'ils s'organisent de façon non homogène et forment des objets de plus grande dimension. $S_{c c}$ est alors reliée à la conformation et à l'organisation de ces nouvelles entités. Nous reviendrons sur ce point particulier dans les exemples présentant les fonctions de structure partielles mesurées par diffusion de neutron.

Les contributions respectives des différentes fonctions de structure partielles sont pondérées par $K_{m}$ et $K_{c}(\mathrm{~cm})$, les longueurs de contraste des monomères $(m)$ et des contreions $(c)$ par rapport au solvant $(s)$. Nous considérerons que chaque monomère diffuse de façon identique, c'est-à-dire qu'il n'y a qu'une seule longueur de contraste $K_{m}$, comme dans le cas des homopolymères. $K_{m}^{2}$ et $K_{c}^{2}\left(\mathrm{~cm}^{2}\right)$ sont les facteurs de contraste. $K_{m}$ et $K_{c}$ sont définies à partir des longueurs de diffusion cohérentes $a(\mathrm{~cm})$ et des volumes molaires $v\left(\mathrm{~cm}^{3} / \mathrm{mol}\right)$ de chacun des constituants. On peut également les écrire en fonction de densités de longueur de diffusion $\rho\left(\mathrm{cm}^{-2}\right)$ :

$$
\left\{\begin{array}{l}
K_{m}=a_{m}-a_{s} \cdot \frac{v_{m}}{v_{s}}=\frac{v_{m}}{N_{a}}\left(\rho_{m}-\rho_{s}\right) \\
K_{c}=a_{c}-a_{s} \cdot \frac{v_{c}}{v_{s}}=\frac{v_{c}}{N_{a}}\left(\rho_{c}-\rho_{s}\right)
\end{array} \text { avec } \rho_{m}=\frac{a_{m}}{v_{m}} N_{a}, \quad \rho_{c}=\frac{a_{c}}{v_{c}} N_{a}, \quad \rho_{s}=\frac{a_{s}}{v_{s}} N_{a} .\right.
$$

En diffusion de neutrons, il est généralement possible d'accéder indépendamment aux fonctions de structure partielles $S_{m m}$ et $S_{c c}$ en faisant «varier le contraste», et en particulier, en travaillant avec des mélanges de solvants H/D qui annulent respectivement $K_{c}$ et $K_{m}$. Pour un mélange de solvants H/D (généralement $\mathrm{H}_{2} \mathrm{O} / \mathrm{D}_{2} \mathrm{O}$ ), la densité de longueur de diffusion s'exprime comme :

$$
\rho_{s}^{\text {mélange }}=\phi_{v}^{H} \cdot \rho_{s}^{H}+\left(1-\phi_{v}^{H}\right) \cdot \rho_{s}^{D}
$$

où $\phi_{v}^{H}$ est la fraction volumique de solvant $H$. Un choix judicieux de $\phi_{v}^{H}$ permet d'égaliser la densité de longueur de diffusion du solvant à celle de l'un des deux constituants (méthode d'effacement du contraste), et donc de le masquer et de révéler la fonction de structure de l'autre composant.

La variation de contraste constitue un avantage important qu'il faut savoir mettre à profit si l'on veut sonder de façon indépendante la structure des polyions $\left(\rho_{s}=\rho_{c}, \Sigma^{c o h}(q)=K_{m}^{2} S_{m m}(q)\right)$ ou celle des contreions $\left(\rho_{s}=\rho_{m}, \Sigma^{c o h}(q)=K_{c}^{2} S_{c c}(q)\right)$. En diffusion de rayons $\mathrm{X}$, on se trouve généralement dans un cas intermédiaire : les trois fonctions de structure contribuent à l'intensité diffusée. On peut toutefois, lorsque c'est possible, essayer de privilégier $S_{c c}$ en travaillant avec des contreions «lourds » $\left(\mathrm{Cs}^{+}\right)$, ou au contraire, $S_{m m}$ avec des contreions «légers $\left(\mathrm{H}^{+} \text {ou } \mathrm{TMA}^{+}\right)^{2}$.

Toutes ces fonctions de structure combinent des corrélations intra et intermoléculaires. Malgré cette difficulté apparente, c'est vers elles que l'on se tourne (ou vers une combinaison de celles-ci lorsque les conditions de contraste ne sont pas maîtrisées) lorsque l'on cherche à caractériser le plus simplement possible l'état de dispersion des différents constituants (organisation intermoléculaire). En effet, comme

\footnotetext{
${ }^{2}$ Une autre possibilité consisterait à utiliser la technique de diffusion anomale avec le rayonnement synchrotron.
} 
nous le verrons par la suite, elles présentent un maximum facilement identifiable caractéristique de l'organisation des différentes espèces en absence de sel ajouté.

Pour préparer une expérience de diffusion, tout comme pour analyser correctement les données, il est important de connaître les densités de longueur de diffusion des différents constituants. Il faut pour cela reconstituer les diffuseurs élémentaires (monomères, contreions et molécules de solvant) à partir des groupements chimiques de base. En considérant l'additivité de leurs volumes molaires partiels et de leurs longueurs de diffusion cohérente ${ }^{3}$, on peut alors recalculer le volume molaire total $v\left(\mathrm{~cm}^{3} / \mathrm{mol}\right)$ ainsi que la longueur de diffusion globale $a(\mathrm{~cm})$ et finalement, la densité de longueur de diffusion $\rho$ $\left(\mathrm{cm}^{-2}\right)$ recherchée $\mathrm{e}^{4}$.

Un problème peut cependant se poser avec les espèces chargées. Les ions ont généralement tendance à s'entourer d'un cortège de molécules de solvant qui s'organisent de façon ordonnée et minimisent l'espace occupé (phénomène d'électrostriction). C'est cette nouvelle entité (ion hydraté) qu'il faut prendre en compte dans le calcul de $\rho$. Les ions condensés et les ions libres peuvent néanmoins se comporter de façon différente vis-à-vis des molécules de solvant, et des doutes peuvent apparaître quant à la bonne valeur de $\rho$ à utiliser. Pour éviter ce genre de problème, on trouve assez souvent dans la littérature, le contreion tétraméthyle ammonium $\left(\mathrm{TMA}^{+}\right)$qui à l'avantage de pas présenter cet effet d'électrostriction.

\subsection{Fonctions de corrélation intra et intermoléculaires}

Lorsque l'on s'intéresse plus particulièrement aux macroions, et que l'on cherche à déterminer proprement leurs fonctions de structure intra (facteur de forme) et intermoléculaire, il est nécessaire d'extraire expérimentalement chacune de ces contributions. Par la suite, nous considèrerons que la contribution des contreions à l'intensité diffusée est négligeable. Comme nous l'avons mentionné précédemment, le calcul de leur densité de longueur de diffusion n'est pas forcément évident. Il doit donc être réalisé avec soin pour valider cette hypothèse.

Dans ces conditions, $\Sigma^{c o h}(q)=K_{m}^{2} S_{m m}(q)$, ou pour simplifier, $\Sigma^{c o h}(q)=K^{2} S(q) . S\left(q, c_{p}\right)$ est souvent appelée fonction de structure totale et notée $S_{T}\left(q, c_{p}\right)$. En distinguant les paires de diffuseurs sur une même chaîne et sur des chaînes différentes, $S\left(q, c_{p}\right)$ (ou $S_{T}\left(q, c_{p}\right)$ ) peut se décomposer en deux termes associés aux corrélations intramoléculaires et intermoléculaires :

$$
S\left(q, c_{p}\right)=S_{T}\left(q, c_{p}\right)=c_{p} \cdot g\left(q, c_{p}\right)=S_{1}\left(q, c_{p}\right)+S_{2}\left(q, c_{p}\right)=c_{p} \cdot g_{1}\left(q, c_{p}\right)+c_{p}^{2} \cdot g_{2}\left(q, c_{p}\right) .
$$

Dans cette expression, $S_{1}\left(q, c_{p}\right)$ et $S_{2}\left(q, c_{p}\right)$ sont les fonctions de structure intra et intermoléculaires $\left(\mathrm{cm}^{-3}\right), g\left(q, c_{p}\right)$ est la fonction de corrélation de paires du formalisme de van Hoove [4], $g_{1}\left(q, c_{p}\right)$ est le facteur de forme (sans unité) de la molécule, et $g_{2}\left(q, c_{p}\right)$ la fonction de corrélation intermoléculaire $\left(\mathrm{cm}^{3}\right) . c_{p}$ est la concentration en nombre en monomères $\left(\mathrm{cm}^{-3}\right)$. Compte tenu du caractère répulsif des interactions interchaînes, $S_{2}\left(q, c_{p}\right)$ et $g_{2}\left(q, c_{p}\right)$ sont des fonctions négatives. Notons que le facteur de forme $g_{1}(q)$ peut également se mettre sous la forme $N P(q)$ où $P(q)$ est le facteur de forme normalisé $(P(q=0)=1)$, et $N$, le degré de polymérisation des chaînes. Le problème consiste désormais à mesurer expérimentalement chacune de ces contributions.

Pour déterminer le facteur de forme $g_{1}(q)$, la méthode la plus simple consiste généralement à faire des mesures à des concentrations suffisamment faibles pour que les corrélations intermoléculaires soient négligeables. On peut également mesurer l'intensité diffusée à plusieurs concentrations et extrapoler $S\left(q, c_{p}\right) / c_{p}$ à concentration nulle $\left(\lim _{c p \rightarrow O} S\left(q, c_{p}\right) / c_{p}=g_{1}\left(q, c_{p}\right)\right)$. Ces méthodes supposent que le facteur de forme ne change pas avec la concentration. Cette méthode n'est donc pas applicable aux

\footnotetext{
3 Aux rayons X, la longueur de diffusion d'un atome est proportionnelle à son nombre d'électrons. Elle est donnée par la relation $a_{X}\left(10^{-13} \mathrm{~cm}\right)=2.82 Z$ où $Z$ est le numéro atomique de l'atome considéré.

${ }^{4}$ On a alors $\rho\left(10^{10} \mathrm{~cm}^{-2}\right)=\frac{a\left(10^{-13} \mathrm{~cm}\right)}{v\left(\mathrm{~cm}^{3} \mathrm{~mol}^{-1}\right)} 6.022\left(10^{23} \mathrm{~mol}^{-1}\right)$.
} 
PEs car la portée des interactions électrostatiques et la conformation moyenne des chaînes varient avec celle-ci.

D'autres méthodes sont heureusement envisageables en utilisant la diffusion de neutrons et la substitution isotopique H/D au niveau du solvant et des polyions. L'une d'entre elles consiste à utiliser un mélange de chaînes $\mathrm{H}$ et $\mathrm{D}$ identiques (de même degré de polymérisation) dans un solvant qui annule la longueur de contraste de l'une des deux espèces. Prenons par exemple un mélange de concentration totale $c_{p}$, contenant une fraction $x$ de chaînes hydrogénées dans un solvant tel que $K_{D}=0$. Dans ces conditions :

$$
\Sigma^{c o h}(q, x)=K_{H}^{2} S_{H H}(q, x)=K_{H}^{2}\left(x . c_{p} \cdot g_{1}\left(q, c_{p}\right)+x^{2} \cdot c_{p}^{2} \cdot g_{2}\left(q, c_{p}\right)\right),
$$

et par conséquent :

$$
\frac{\Sigma^{c o h}(q, x)}{x \cdot c_{p} \cdot K_{H}^{2}}=g_{1}\left(q, c_{p}\right)+x . c_{p} \cdot g_{2}\left(q, c_{p}\right) .
$$

Il suffit, pour une concentration $c_{p}$ donnée, de réaliser des mesures à différentes valeurs de $x$ et d'extrapoler la relation précédente à $x=0$ pour obtenir le facteur de forme recherché. Connaissant $g_{1}\left(q, c_{p}\right)$ et $\Sigma^{c o h}(q, x)$, on peut alors remonter à $g_{2}\left(q, c_{p}\right)$.

La méthode du contraste moyen nul (ZAC ou «Zero Average Contraste »en anglais) permet d'obtenir $g_{1}\left(q, c_{p}\right)$ en une seule mesure. Elle est également basée sur l'utilisation d'un mélange de chaînes H et D identiques. La section efficace cohérente s'écrit :

$$
\Sigma^{c o h}\left(q, c_{p}\right)=K_{H}^{2} S_{H H}\left(q, c_{p}\right)+K_{D}^{2} S_{D D}\left(q, c_{p}\right)+2 K_{H} K_{D} S_{H D}\left(q, c_{p}\right) .
$$

Si les chaînes $\mathrm{H}$ et $\mathrm{D}$ ont le même degré de polymérisation, leur facteur de forme $g_{1}\left(q, c_{p}\right)$ ainsi que leur fonction de corrélation intermoléculaire $g_{2}\left(q, c_{p}\right)$ sont identiques. Les fonctions structure partielles s'expriment alors comme :

$$
\left\{\begin{array}{l}
S_{H H}\left(q, c_{p}\right)=x \cdot c_{p} \cdot g_{1}\left(q, c_{p}\right)+x^{2} \cdot c_{p}^{2} \cdot g_{2}\left(q, c_{p}\right) \\
S_{D D}\left(q, c_{p}\right)=(1-x) \cdot c_{p} \cdot g_{1}\left(q, c_{p}\right)+(1-x)^{2} \cdot c_{p}^{2} \cdot g_{2}\left(q, c_{p}\right) \\
S_{H D}\left(q, c_{p}\right)=x \cdot(1-x) \cdot c_{p}^{2} \cdot g_{2}\left(q, c_{p}\right) .
\end{array}\right.
$$

En introduisant chacune d'entre elles dans la relation précédente, on obtient :

$$
\Sigma^{c o h}\left(q, c_{p}\right)=\left[x \cdot K_{H}^{2}+(1-x) \cdot K_{D}^{2}\right] \cdot c_{p} \cdot g_{1}\left(q, c_{p}\right)+\left[x \cdot K_{H}+(1-x) \cdot K_{D}\right]^{2} \cdot c_{p}^{2} \cdot g_{2}\left(q, c_{p}\right) .
$$

Pour annuler les corrélations intermoléculaires $g_{2}(q)$, il suffit que le contraste moyen du mélange $\left[x \cdot K_{H}+(1-x) \cdot K_{D}\right]$ soit nul. On choisit généralement $x=0.5$, ce qui impose $K_{H}=-K_{D}=K$. On aboutit alors à :

$$
\begin{aligned}
& \Sigma^{c o h}\left(q, c_{p}\right)=K^{2} \cdot c_{p} \cdot g_{1}\left(q, c_{p}\right) \\
& g_{1}\left(q, c_{p}\right)=\frac{\Sigma^{c o h}\left(q, c_{p}\right)}{K^{2} \cdot c_{p}} .
\end{aligned}
$$

La connaissance de $g_{1}\left(q, c_{p}\right)$ (ou de $S_{1}\left(q, c_{p}\right)$ ) ainsi que $g\left(q, c_{p}\right)$ de (ou de $S\left(q, c_{p}\right)$ ) permet de remonter à la fonction de corrélation intermoléculaire $g_{2}\left(q, c_{p}\right)\left(\right.$ ou $\left.S_{2}\left(q, c_{p}\right)\right)$ :

$$
\begin{aligned}
& S_{2}\left(q, c_{p}\right)=S\left(q, c_{p}\right)-S_{1}\left(q, c_{p}\right) \\
& g_{2}\left(q, c_{p}\right)=S_{2}\left(q, c_{p}\right) / c_{p}^{2}=\left(g\left(q, c_{p}\right)-g_{1}\left(q, c_{p}\right)\right) / c_{p} .
\end{aligned}
$$

\subsection{Limite thermodynamique $(q=0)$}

Si l'on considère un système binaire (particule - solvant) constitué de $n$ particules identiques dans un volume $V$ (comme des chaînes de degré de polymérisation $N$ ), la limite thermodynamique $(q=0)$ de 
la fonction de structure $S(q)$ s'écrit $[5,6]$ :

$$
S(q=0)=k_{B} T c_{p}\left(\frac{\partial \pi}{\partial c_{p}}\right)^{-1}=k_{B} T c_{p}^{2} \chi_{T}
$$

où $c_{p}$ est la concentration en nombre en monomères, $\pi$ est la pression osmotique et $\chi_{T}$ est la compressibilité osmotique. En absence d'interaction, $S(q)$ ne représente que la fonction de structure intramoléculaire des chaînes. Comme $\pi / k_{B} T=c_{p} / N$, la limite à $q=0$ est alors égale à $S(q=0)=$ $c_{p} g(q=0)=c_{p} N$. Elle nous renseigne sur le degré de polymérisation des chaînes en solution. Lorsque les interactions sont maximales, comme dans le cas d'un ordre cristallin, la compressibilité osmotique s'annule et par conséquent, $S(q=0)=0$. Pour des interactions répulsives intermédiaires (comme avec des macroions en solution), la valeur de $S(q=0)$ est comprise entre ces deux cas limites. Les plus grands vecteurs de diffusion étant dominés par la fonction de structure intramoléculaire (qui tend vers 0 lorsque pour les plus grandes valeurs de $q$ ), la fonction de structure $S(q)$ peut présenter un maximum pour une certaine valeur $q^{*}$.

Dans le cas des solutions de PEs (chaînes, contreions, solvant), la pression osmotique est dominée par les contreions libres. Il est donc théoriquement possible de tirer une information sur le processus de condensation en analysant l'intensité diffusée à $q=0$. En pratique, cette intensité est généralement difficile à extraire, et les résultats restent souvent qualitatifs. En effet, comme nous le verrons en 5.1, les très faibles vecteurs de diffusion sont souvent entachés d'une remontée parasite qui gêne ce type d'observation.

La présence d'un maximum dans la fonction de structure est effectivement observée expérimentalement pour les solutions de PE en absence de sel ajouté. Si l'origine de celui-ci est de nature électrostatique et reste liée aux répulsions interchaînes, son interprétation physique est cependant délicate. Il peut en effet tout aussi bien provenir d'un ordre de position, que d'un trou de corrélation dans un système isotrope désordonné.

\section{SECTION EFFICACE DIFFÉRENTIELLE DE DIFFUSION COHÉRENTE PAR UNITÉ DE VOLUME}

Cette partie présente des courbes de diffusion mesurées pour différents systèmes en fonction de la concentration $c_{p}$, de la fraction de charge chimique $f$, et des facteurs de contraste. Nous allons essayer de faire ressortir les principales caractéristiques observées et présenter les informations que l'on peut en tirer au regard de certaines approches théoriques. Sauf cas particulier, les longueurs de contraste des monomères et des contreions ne sont pas nulles. La fonction de diffusion mesurée $\Sigma^{c o h}(q)$ est alors une combinaison linéaire des trois fonctions de structure partielles $S_{m m}, S_{c c}$ et $S_{m c}$ qui reflètent elles-mêmes, les corrélations intra et intermoléculaires des différent constituants. Pour une analyse plus détaillée, il peut s'avérer utile de mesurer indépendamment les fonctions de structure partielles $S_{m m}$ et $S_{c c}$. Cela peut s'effectuer par diffusion de neutron en travaillant dans des mélanges de solvants H/D adaptés. Ce type de mesure est généralement réalisé pour caractériser l'organisation intermoléculaire (état de dispersion). Nous verrons que dans certains cas, elles peuvent également nous aider à comprendre la conformation du cortège de contreions condensés autour des chaînes.

\subsection{Effet de la concentration}

Les mesures expérimentales de $\Sigma^{c o h}(q)$ font apparaître un maximum à une position $q^{*}$ qui se déplace vers les plus grands vecteurs de diffusion lorsque l'on augmente la concentration [7-9]. Ce pic, souvent appelé «pic polyélectrolytes », est caractéristique des solutions de polymères chargés et de la présence de répulsions électrostatiques. Les polymères neutres, tout comme les solutions de PEs avec sel ajouté (qui écrante les interactions) ne montrent aucune caractéristique similaire. En régime dilué, l'évolution de la position du pic suit une loi de puissance en $c_{p}^{1 / 3}$. Il traduit l'existence d'un ordre liquide entre 


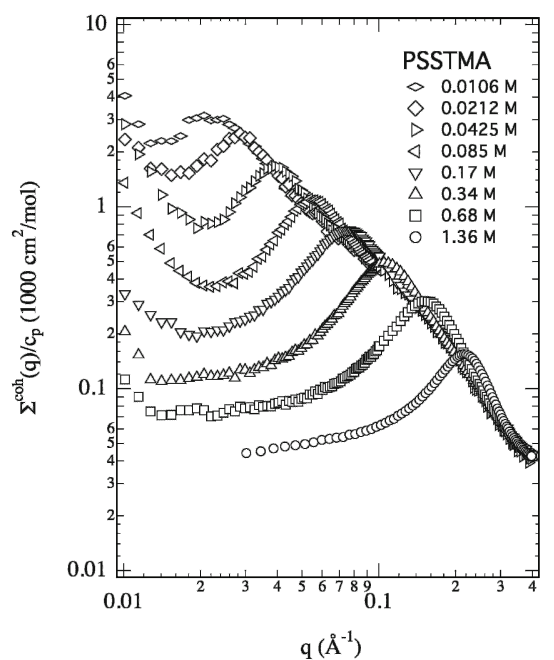

a)

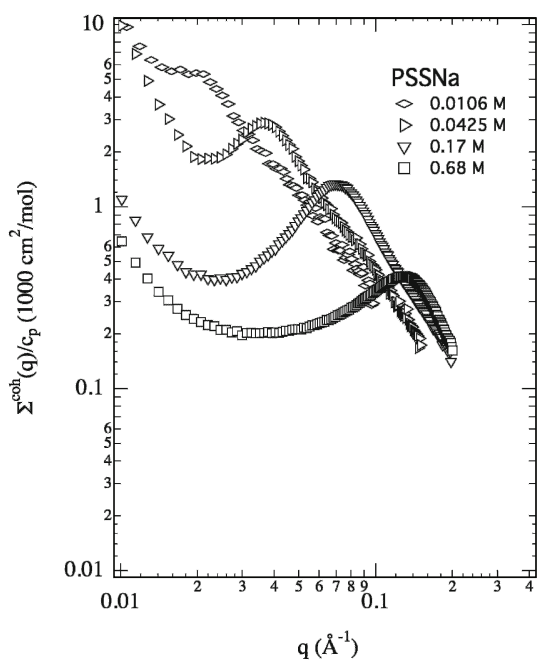

b)

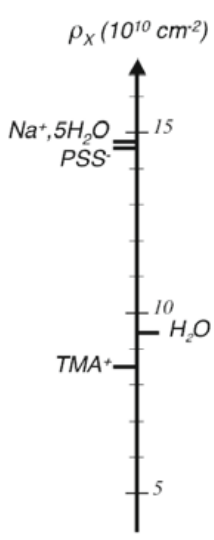

c)

Figure 3. $\Sigma^{c o h}(q) / c_{p}$ mesurées par diffusion de rayons X (ligne D2AM, ESRF, Grenoble) sur des solutions aqueuses de a) PSSTMA et b) PSSNa à différentes concentrations. Dans ces systèmes, $f=1, N=700, \zeta=2.83$. c) Densités de longueur de diffusion des différents constituants pour la diffusion des rayons X. Pour le contreion sodium $\mathrm{Na}^{+}$, la valeur indiquée correspond à celle de l'ion hydraté $\left(\mathrm{Na}^{+}, 5 \mathrm{H}_{2} \mathrm{O}\right)$.

les centres de masse des macromolécules [10]. Lorsque l'on passe en régime semidilué, les chaînes commencent à s'interpénétrer. Le pic est toujours présent, mais son évolution suit désormais une loi en $c_{p}^{1 / 2}$. La transition entre ces deux régimes permet généralement de déterminer la concentration critique de recouvrement $c_{p}^{*}[8,11]$. Ceci n'est toutefois envisageable que pour des chaînes assez courtes sans quoi les concentrations du régime dilué deviennent extrêmement faibles et les intensités diffusées difficilement mesurables. Pour des solutions concentrées (plusieurs mol /L), d'autres phénomènes peuvent également apparaître [12]. Ils ne seront pas discutés dans cette contribution. L'origine et la signification physique du pic de diffusion en régime semidilué seront discutées au paragraphe 5.3.

Deux séries de courbes normalisées à la concentration $\left(\Sigma^{c o h}\left(q, c_{p}\right) / c_{p}\right)$ sont présentées sur la figure 3. Elles ont été obtenues par diffusion des rayons X (ligne D2AM, ESRF, Grenoble), en régime semidilué sur des échantillons de polystyrène sulfonate en présence de contreions tétramethyle ammonium (PSSTMA) et de contreions sodium (PSSNa). Chaque monomère porte une charge négative $(f=1)$, nous sommes en présence de systèmes fortement chargés. Le paramètre de charge $\zeta(=2.83)$ étant supérieur à l'unité, une partie des contreions se condense. La charge effective est alors proche de 0.36, soit en moyenne une charge (effective) tous les trois monomères.

Dans le système PSSTMA, la densité de longueur de diffusion des contreions $\mathrm{TMA}^{+}$est relativement proche de celle du solvant (figure 3(c)). La longueur de contraste correspondante $K_{c}$ est alors faible devant celle du monomère $K_{m}$. Nous sommes dans un cas particulier où la section efficace $\Sigma^{c o h}(q)$ mesurée par rayons X est essentiellement reliée à la fonction de structure partielle des monomères $S_{m m}$. Dans le système PSSNa, ceci n'est plus le cas, $\Sigma^{c o h}(q)$ résulte d'une combinaison linéaire des différentes fonctions de structure partielles, on ne peut plus négliger la contribution des contreions.

Les courbes de diffusion observées pour ces deux systèmes sont assez similaires. Elles montrent clairement un pic de diffusion à une position $q^{*}$ qui se déplace vers les plus grandes valeurs de $q$ lorsque l'on augmente la concentration. Les différences rencontrées au niveau des intensités pour une même concentration sont liées aux longueurs de contraste des différents constituants. 


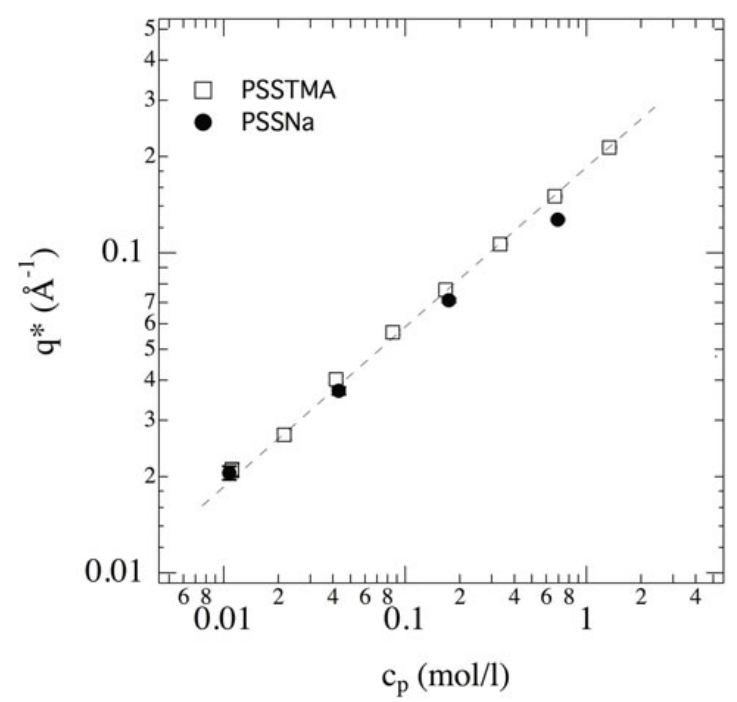

Figure 4. Évolution de la position du maximum $q^{*}$ avec la concentration pour PSSTMA et PSSNa. La ligne en pointillée correspond à une évolution en $c_{p}^{1 / 2}$.

Une observation intéressante concerne l'allure générale des courbes de diffusion dans PSSTMA. Celles-ci tendent en effet à se superposer pour des valeurs de $q$ situées au-delà de la position du maximum $q^{*}$. Dans le cas de PSSTMA, $\Sigma^{c o h}(q)$ est très proche de la fonction de structure des chaines $S_{m m}$. Par conséquent :

$$
\Sigma^{c o h}\left(q, c_{p}\right) / c_{p}=K_{m}^{2}\left(g_{1}\left(q, c_{p}\right)+c_{p} \cdot g_{2}\left(q, c_{p}\right)\right) .
$$

Aux plus grandes valeurs de $q$, on sonde la structure locale des solutions, la contribution de la fonction de corrélation intermoléculaire $g_{2}\left(q, c_{p}\right)$ commence à diminuer, le signal observé se rapproche du facteur de forme des polyions. La superposition des courbes de diffusion (visible uniquement lorsque les intensités sont normalisées à la concentration) traduit le fait que la conformation locale des chaînes (à une échelle spatiale $\left.<2 \pi / q^{*}\right)$ ne varie que très peu avec la concentration.

Pour chacun des composés, l'évolution de $q^{*}$ en fonction de la concentration (figure 4) peut être décrite par une loi de puissance en $c_{p}^{1 / 2}$. Cette évolution est caractéristique des PEs hydrophiles, ou plus exactement, des PEs pour lesquels les principales interactions sont électrostatiques. Lorsque la chaîne présente un caractère hydrophobe, on s'écarte progressivement de ce comportement [13]. Différents régimes en concentration peuvent également apparaître [14]. Il est assez courant dans la littérature de trouver des variations en loi de puissance pour lesquelles l'exposant est légèrement inférieur à $1 / 2$ (proche de 0.45 ). Ce phénomène ne signifie pas pour autant que le système présente d'autres interactions que les simples répulsions électrostatiques. Il peut en effet s'expliquer par la combinaison des différentes fonctions de structure partielles, plus particulièrement lorsqu'une partie des contreions est condensée [15]. Sur les courbes de PSSTMA présentées, les contreions ne contribuent pas, l'exposant est égal à $1 / 2$, aux erreurs expérimentales près. Pour PSSNa, l'exposant reste légèrement inférieur. Le rôle des contreions sur les courbes de diffusion peut être appréhendé de façon plus précise lorsque l'on mesure séparément les fonctions de structure partielle $S_{m m}$ et $S_{c c}$ d'un même composé. Nous aborderons ce problème dans le prochain paragraphe.

Au-delà de la présence du pic de diffusion, on observe également une remontée aux plus petits vecteurs de diffusion. Cette propriété particulière est très souvent observée dans les solutions de PEs [16]. Elle se manifeste en diffusion dynamique de la lumière par l'apparition d'un mode «lent» et traduit la présence de domaines de très grandes dimensions dans le système. Cette diffusion peut gêner 


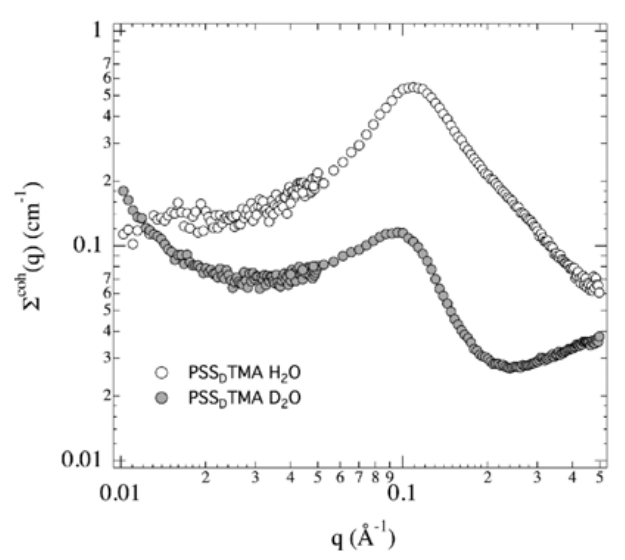

a)

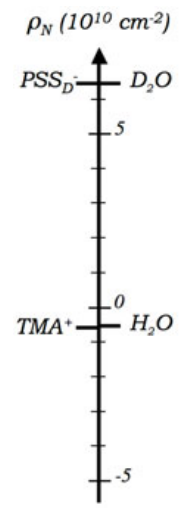

b)

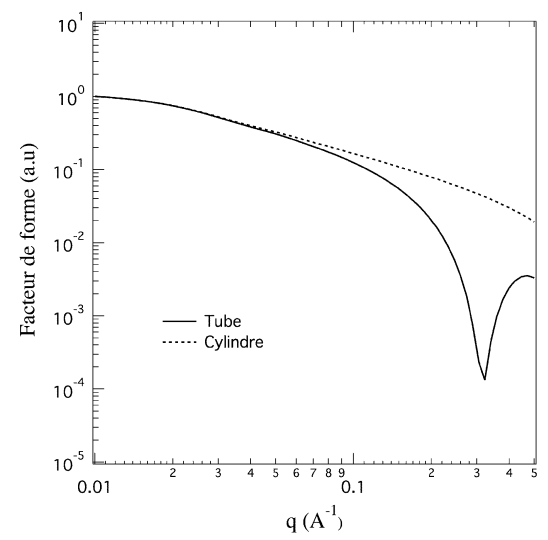

c)

Figure 5. a) $\Sigma^{c o h}(q)$ mesurées par diffusion de neutron (D22, ILL, Grenoble) sur le système polystyrène sulfonate $\mathrm{D} /$ tétraméthyle ammonium $\mathrm{H}$ totalement chargé $(f=1)$ à $c_{p}=0.34 \mathrm{~mol} / \mathrm{L}$ dans l'eau hydrogénée $\left(\mathrm{PSS}_{D} \mathrm{TMA}^{2}\right.$ $\mathrm{H}_{2} \mathrm{O}$ ) et deutériée $\left(\operatorname{PSS}_{D}\right.$ TMA $\left.\mathrm{D}_{2} \mathrm{O}\right)$. Dans $\mathrm{H}_{2} \mathrm{O}, \Sigma^{c o h}(q)=\mathrm{K}_{m}^{2} S_{m m}(q)$. Dans $\mathrm{D}_{2} \mathrm{O}, \Sigma^{c o h}(q)=\mathrm{K}_{c}^{2} S_{c c}(q)$. b) densité de longueur de diffusion des différents constituants pour la diffusion de neutrons. c) facteur de forme théorique (en unités arbitraires) pour un cylindre (rayon : $5 \AA$, longueur : $200 \AA$ ), et pour un tube (rayon intérieur : $5 \AA$, rayon extérieur : $10 \AA$, longueur : $200 \AA$ ).

considérablement l'analyse des données, en particulier lorsque l'information recherchée se situe aux très faibles vecteurs de diffusion. Nous n'irons toutefois pas plus loin dans le détail de ces remontées (« upturns » en anglais) si ce n'est pour souligner l'importance de la préparation des solutions de PE.

\subsection{Effet du contraste : fonctions de structure partielles}

Les courbes de diffusion sont reliées à l'organisation spatiale des deux constituants : polyions et contreions. Pour extraire des informations sur leur organisation respective, il est nécessaire de mesurer les fonctions de structure partielles $S_{\mathrm{mm}}$ et $S_{\mathrm{cc}}$. Cela peut se faire proprement en diffusion de neutron en utilisant l'effacement de contraste. Il faut alors adapter la densité de longueur de diffusion du solvant à celle du monomère (mesure de $S_{\mathrm{cc}}$ ) ou à celle du contreion (mesure de $S_{\mathrm{mm}}$ ) grâce à des mélanges $\mathrm{H}_{2} \mathrm{O} / \mathrm{D}_{2} \mathrm{O}$ adaptés.

L'exemple présenté sur la figure 5(a) (D22, ILL, Grenoble) correspond à des mesures réalisées par diffusion de neutrons sur le même système que précédemment (PSSTMA). Il était toutefois composé de chaînes de $\mathrm{PSS}^{-}$deutériées $\left(\mathrm{PSS}_{D}^{-}\right)$et de contreions tétraméthyle ammonium hydrogénés $\left(\mathrm{TMA}^{+}\right)$. Dans ces conditions, $\rho_{D_{2} O}=\rho_{P S S_{D}^{-}}$et $\rho_{H_{2} O}=\rho_{T M A+}$ (figure 5(b)). Une mesure dans $\mathrm{D}_{2} \mathrm{O}$ nous fournit donc $S_{c c},\left(\Sigma^{c o h}(q)=K_{c}^{2} S_{c c}(q)\right)$, tandis qu'une mesure dans $\mathrm{H}_{2} \mathrm{O}$ permet de déterminer $S_{m m}\left(\Sigma^{c o h}(q)=K_{m}^{2} S_{m m}(q)\right)$.

Les courbes mesurées par diffusion de neutrons permettent de mettre en évidence de nouveaux éléments et complètent l'analyse précédente réalisée aux rayons $\mathrm{X}$.

L'observation principale concerne la présence d'un pic de diffusion sur le signal des contreions $\left(S_{c c}\right)$. Il apparaît à une position quasiment identique à celle observée sur le signal des chaînes $\left(S_{m m}\right)$ (nous reviendrons sur cette petite variation en fin de paragraphe). Cela signifie que les contreions ne sont pas distribués de façon homogène dans la solution. Il s'agit là d'une manifestation directe du phénomène de condensation. Les contreions se positionnant autour des chaînes, ils créent des gaines qui reproduisent l'organisation spatiale des polyions. Il apparaît donc un maximum dans la fonction de structure partielle 
à une position très voisine de celle rencontrée dans $S_{m m}$. En absence de condensation, on s'attendrait à une fonction de structure $S_{c c}$ complètement plate dans ce domaine de vecteurs de diffusion.

Un autre point important concerne la présence d'une remontée vers $0.4-0.5 \AA^{-1}$ dans $S_{c c}$ qui n'apparaît pas sur la fonction de structure des chaînes $S_{m m}$. Si les polyions s'apparentent à des cylindres de quelques $\AA$ de diamètre, les contreions condensés forment des tubes qui les enrobent. La fonction de structure partielle $S_{c c}$ met donc en jeu une contribution intra (facteur de forme) et inter associées à ces nouvelles entités. Aux plus grands vecteurs de diffusion, on peut admettre que la principale contribution à l'intensité diffusée provient du facteur de forme des objets diffusants. Les facteurs de forme d'un tube et d'un cylindre sont représentés sur la figure 5(c). La première oscillation du tube apparaît à plus petit vecteur de diffusion que celle d'un cylindre classique (qui sort de la gamme accessible). Elle peut donc expliquer la remontée observée dans la fonction de structure $S_{c c}$. En réalité, le calcul exact du facteur de forme associé aux gaines de contreions est assez complexe à calculer car ils ne forment pas une paroi de densité constante. Pour le déterminer de façon précise, il faut connaître leur profil de concentration autour des polyions. Une première approximation consiste à résoudre l'équation de Poisson-Boltzmann dans un modèle de cellule cylindrique. Si cette approche permet de calculer la contribution du facteur de forme au facteur de structure partiel, il ne permet évidemment pas de reproduire le pic de diffusion observé [17-19].

La position du maximum observé dans les fonctions de structure partielles $S_{m m}$ et $S_{c c}$ est légèrement différente. Cet effet est toutefois d'autant plus important que la concentration est élevée. Il montre que $q^{*}$ est sensible à la nature de l'espèce diffusante. Lorsque la diffusion est dominée par les contreions condensés, la position du pic est déplacée aux plus petites valeurs de $q$. En réalité, chaque fonction de structure partielle est la somme d'une contribution intra et intermoléculaire. La position du pic de diffusion peut être légèrement affectée si l'intra est modifiée. La différence entre les facteurs de forme des chaînes et des contreions condensés évoquée précédemment (figure 5(c)) peut donc expliquer ces petites variations.

Ce phénomène peut être à l'origine des écarts souvent rencontrés expérimentalement dans les lois de puissance reliant $q^{*}$ à $c_{p}$ (où l'exposant mesuré est légèrement inférieur à $1 / 2$ ). Il confirme en tout cas les différences observées en diffusion de rayons X sur les systèmes PSSTMA et PSSNa.

\subsection{Effet du taux de charge}

L'évolution des courbes de diffusion avec la fraction de charge chimique du polyion ( $f$ ) est un point important à étudier si l'on veut comprendre la physique de ces systèmes. Ce type d'étude est curieusement assez difficile à trouver dans la littérature, la synthèse chimique des composés s'avérant souvent délicate. Afin de pouvoir interpréter correctement les données, le choix du PE doit être réalisé avec soins. Pour faire varier le taux de charge des PEs forts, il faut synthétiser des copolymères statistiques formés de monomères ionisables et de monomères neutres. La difficulté principale est liée au fait que les monomères neutres ne doivent pas introduire d'interaction hydrophobe dans le système. En d'autres termes, les monomères non chargés doivent présenter un caractère hydrophile marqué, sans quoi, en abaissant le taux de charge, on augmente également l'hydrophobie des chaînes. Lorsque cela n'est pas le cas, on note des modifications importantes du facteur de forme des macromolécules qui peuvent conduire à des conformations particulières en collier de perles [14, 20] et à des écarts importants à la loi $q^{*} \propto c_{p}^{1 / 2}$. Pour cette raison, le polystyrène sulfonate (PSS) dans lequel une partie des monomères ionisables est remplacée par des monomères de polystyrène neutres (PS) n'est pas un candidat idéal $[13,21]$. Une autre difficulté majeure est d'obtenir une distribution statistique des différents monomères le long de la chaîne afin d'éviter la formation de blocs ionisables et de blocs neutres.

Les travaux pionniers ont été réalisés sur des solutions de copolymères acrylamide-acide acrylique [22] et sur des solutions d'alcool polyvinylique partiellement estérifié (NaPVS) [23]. Pour chaque fraction de charge $f$, le pic de diffusion est toujours présent et montre une évolution caractéristique en $c_{p}^{1 / 2}$. L'évolution de $q^{*}$ en fonction de $f$ est présentée sur la figure 6 pour différentes concentrations 


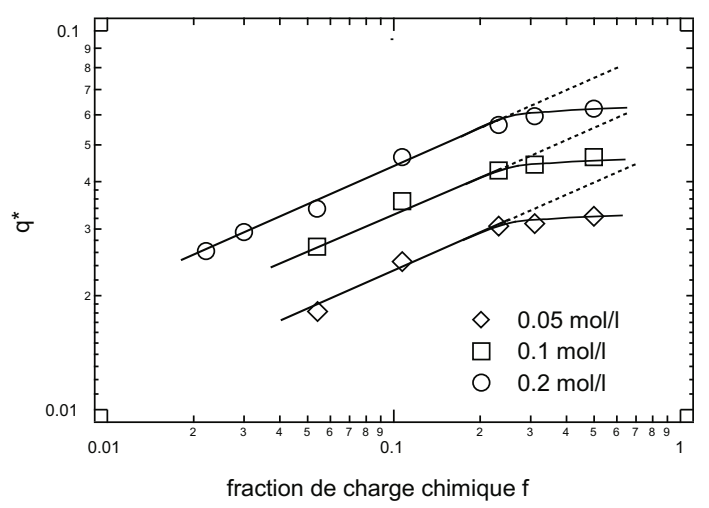

Figure 6. Évolution de la position du pic de diffusion $q^{*}$ en fonction de la fraction de charge chimique $f$ portée par le polyion (NaPVS) [23] pour trois concentrations différentes. Le trait plein correspond à une évolution en loi de puissance $q^{*} \propto f^{\alpha}$ avec $\alpha=0.3$. Les mesures ont été réalisées par diffusion des rayons $\mathrm{X}$.

en NaPVS. Lorsque l'on augmente le taux de charge du polyion, le pic de diffusion est déplacé vers les plus grandes valeurs de $q$. Dans ce régime, l'évolution de $q^{*}$ suit une loi de puissance en $f^{\approx 0.3}$. Au-delà d'une certaine charge critique, sa position ne varie plus. Cette valeur particulière étant proche du seuil de Manning Oosawa $(f=0.36, \zeta=1)$, elle semble corrélée au phénomène de condensation des contreions qui conduit à une renormalisation de la charge ( $f_{e f f}=$ constante). En d'autres termes, malgré l'augmentation du nombre de groupements chargés le long de la chaîne, la charge effective portée par le polyion, tout comme la position du pic restent constants. Ce phénomène a depuis été confirmé par des études sur le poly(acrylamide- co-sodium-2-acrylamido-2-methylpropane sulfonate (AMAMPS) [24,25]. Dans ce composé, des mesures réalisées par diffusion de neutrons pour différents taux de charge $f$ au-delà du seuil de condensation $(\zeta>1)$ ont permis de déterminer la fonction de structure partielle associée aux seuls macroions. Il en ressort une très grande similitude des fonctions de diffusion indiquant une structure des chaînes quasi-identique à charge effective constante.

De ces études ressort une propriété importante : la position du maximum de diffusion est directement reliée à la fraction de charge nette portée par les macroions. Pour les taux de charge les plus faibles, elle est égale à la fraction de charge chimique $f$ portée par les chaînes. Pour les taux de charge les plus forts, elle correspond à la fraction de charge effective $f_{\text {eff }}$ résultant de la condensation d'une partie des contreions. Il est donc possible de sonder le phénomène de condensation (en absence de transition conformationnelle) en suivant la seule évolution de $q^{*}$.

Cette propriété peut être mise à profit pour étudier la renormalisation de charge dans des solutions de $\operatorname{PSS}^{-}(f=1)$ en présence d'un mélange de contreions monovalents $\left(\mathrm{Na}^{+}\right)$et divalents $\left(\mathrm{Ca}^{2+}\right)[15,26]$. Dans les expériences réalisées, la fraction en ions monovalents $(X)$ varie de $X=0$ (contreions tous divalents $\mathrm{Z}=2, \zeta=2.83>1 / 2, f_{\text {eff }}=0.18$ ) à $X=1$ (contreions tous monovalents, $\zeta=2.83>1$, $\left.f_{e f f}=0.35\right)$. Pour des fractions intermédiaires $(0<X<1)$, la fraction de charge effective finale $f_{\text {eff }}$ résulte d'une subtile compétition entre les processus de condensation des contreions monovalents et divalents. Suivant la valeur de $X$, il est possible de faire varier, non pas la fraction de charge chimique (fixée lors de la synthèse) comme dans l'exemple précédent, mais la fraction de charge effective. Celleci peut être déterminée à partir de l'approche de Manning-Oosawa, ou en résolvant numériquement l'équation de Poisson Boltzmann (Figure 7(a)). En utilisant la loi de puissance $q^{*} \propto f^{0.3}$ précédente, on peut alors comparer les valeurs théoriques de $q^{*}$ avec celles mesurées expérimentalement par diffusion de rayon $X$ (figure 7(b)).

Si la position du pic était reliée à la seule fraction de charge chimique des polyions, celle-ci ne devrait pas bouger avec la valence des contreions. Ce n'est pas ce qu'on observe expérimentalement. Pour les grandes valeurs de $X(\mathrm{X}>0.6$ dans l'approche $\mathrm{PB})$, la charge effective est quasiment constante. Dans 


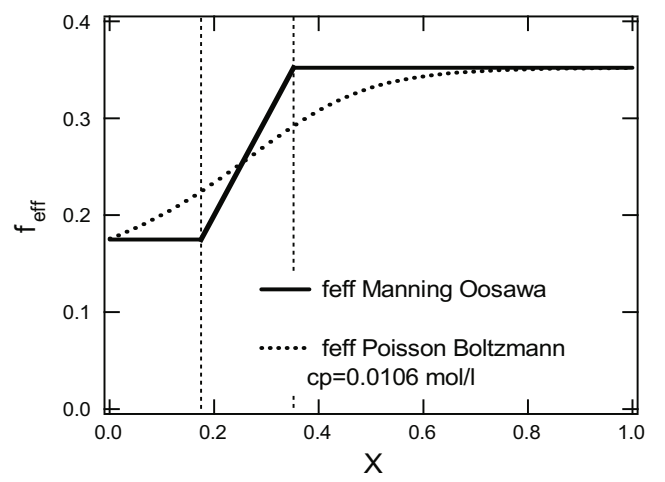

a)

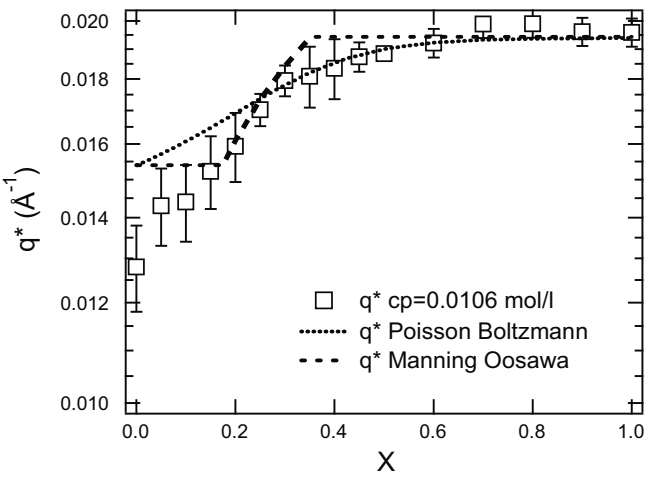

b)

Figure 7. a) Charge effective en fonction de $X$ d'après Manning Oosawa, et après résolution de l'équation de Poisson Boltzmann $\left(c_{p}=0.0106 \mathrm{~mol} / \mathrm{L}\right)$. b) Evolution de la position du pic de diffusion $q^{*}$ en fonction de la fraction en contreions monovalents $X$ et valeurs théoriques déduites de la charge effective prédite par Manning Oosawa et Poisson Boltzmann. Les expériences ont été réalisées par diffusion des rayons $\mathrm{X}$ aux petits angles (ligne D2AM, ESRF, Grenoble) [15].

le même temps, la position du maximum reste inchangée. Lorsque $f_{\text {eff }}$ diminue, cela s'accompagne également d'une diminution de $q^{*}$. Ces observations démontrent à nouveau la corrélation entre ces deux grandeurs. La description théorique du phénomène de condensation rend compte des mesures expérimentales tant que les fractions en ions monovalents ne sont pas trop faibles $(\mathrm{X}>0.2)$. Dans cette région, l'évolution de la position du maximum, et donc, la structure des solutions, peut se comprendre sur la base d'une simple variation de charge effective. Pour les fractions les plus faibles ( $\mathrm{X}>0.2$ ), la proportion d'ions divalents (libres et condensés) devient importante et modifie également la conformation moyenne des macroions (ponts électrostatiques). Ce phénomène va au-delà d'une simple renormalisation de la charge.

\subsection{Vers une description universelle ?}

Si l'origine du pic de diffusion est de nature électrostatique et liée aux répulsions interchaînes, son interprétation physique reste cependant délicate. Elle pourrait être reliée à un ordre cristallin ou de position, dans lequel les chaînes (ou des parties de celles-ci) s'arrangent parallèlement les unes par rapport aux autres. Dans cet ordre bidimensionnel hexagonal, la position du pic est donnée par la distance inter-réticulaire, et son évolution avec la concentration résulte d'une simple loi de dilution. On peut cependant noter qu' aucun pic d'ordre supérieur n'a jamais été observé expérimentalement dans ce genre de solutions.

Le maximum peut également provenir de l'existence d'un «trou de corrélation »autour de chaque polyion, avec un organisation isotrope désordonnée. Dans cette approche, les chaînes ne peuvent se rapprocher au-delà d'une certaine distance caractéristique de l'ordre de la longueur d'écran de Debye. Elles forment un réseau temporaire désordonné de taille $\xi$. Une telle solution donne lieu en diffusion de rayonnement à un maximum dont la position $q^{*}$ est directement reliée à la distance minimale d'approche entre les brins des différents macroions $\left(q^{*}=2 \pi / \xi\right)[10,27]$.

Pour chacune de ces deux approches, l'évolution de la position du pic en fonction de la concentration est similaire et varie en $c_{p}^{1 / 2}$. Il est souvent difficile de trancher définitivement pour l'une ou l'autre de ces descriptions.

La position du pic de diffusion $q^{*}$ traduit donc l'organisation spatiale ou l'état de dispersion des chaînes dans la solution sous l'effet des répulsions électrostatiques. Si sa dépendance avec la 
concentration et la charge nette ne fait plus aucun doute, elle peut en toute logique, également varier avec d'autres paramètres, comme la taille du monomère, la nature du solvant (via la longueur de Bjerrum $\left.l_{B}\right)$... La figure 8a présente l'évolution de $q^{*}$ en fonction de la concentration pour différents PEs, à différents taux de charge (de part et d'autre du seuil de condensation), dans différents solvants, en présence de contreions monovalents [15]. Si toutes les évolutions présentées sont bien en $c_{p}^{1 / 2}$, on note une grande dispersion des valeurs expérimentales. La seule concentration n'est donc pas un paramètre adapté pour décrire de façon universelle la position $q^{*}$ dans ces différents systèmes.

La dépendance de $q^{*}$ avec tous les paramètres du système reste encore de nos jours un défi à surmonter. La conformation moyenne des polyions en solution et leur état de dispersion sont extrêmement difficiles à décrire d'un point de vue théorique. Dans les approches de type lois d'échelles développées initialement pour les chaînes faiblement chargées [10, 28] (repris récemment par Dobrynin et col. [29]), le polyion est composé de blobs électrostatiques de taille $\xi_{e}$. À l'intérieur de ces blobs, la chaîne n'est pas perturbée par la présence des charges et adopte une conformation identique à celle d'une chaîne neutre. À une échelle plus grande, les répulsions électrostatiques l'emportent, les blobs se repoussent et s'alignent. En régime dilué, la chaîne est donc globalement étendue. En régime semidilué, les chaînes commencent à s'interpénétrer et forment un réseau temporaire (modèle isotrope [10]). Le modèle postule l'existence d'une seule longueur caractéristique $\xi$ qui décrit tout le système. Cette longueur représente à la fois la maille du réseau, mais également la portée des interactions électrostatiques. Pour des échelles inférieures à $\xi$, la chaîne adopte la conformation étirée du régime dilué. Pour des échelles supérieures, les interactions électrostatiques sont écrantées. Elle forme une marche aléatoire de blobs de corrélation de taille $\xi$. Une représentation schématique de la structure est présentée sur la figure 9.

L'analyse en loi d'échelle permet finalement de calculer la longueur de corrélation $\xi$. En bon solvant, elle est donnée par:

$$
\xi=f^{-2 / 7}\left(l_{B} / b\right)^{-1 / 7}\left(b c_{p}\right)^{-1 / 2}
$$

La position du maximum $q^{*}$ est alors reliée à l'inverse de cette longueur caractéristique et prend comme expression :

$$
q^{*}=\frac{2 \pi}{\xi}=2 \pi \mathrm{f}^{2 / 7}\left(l_{B} / b\right)^{1 / 7}\left(b c_{p}\right)^{1 / 2} .
$$

Cette approche rend compte des principaux résultats expérimentaux observés. Elle prédit non seulement une variation en $c_{p}^{1 / 2}$, mais également une évolution en $f^{\approx 0.3}(2 / 7=0.285)$. Cette dernière est en fait reliée au changement de taille du blob électrostatique, et finalement, à un changement de longueur de contour effectif de la macromolécule. La position du pic est donc liée à la structure «locale »du polyion. Dans le cas des PEs hydrophobes qui présentent une conformation radicalement différente (dite en collier de perles), son évolution avec la concentration et le taux de charge, est complètement différente [14].

On dispose désormais d'une expression théorique qu'il est facile de comparer à l'ensemble des mesures présentées sur la figure 8. Lorsque l'on calcule la longueur de corrélation électrostatique théorique $\xi$ pour chacun des systèmes présentés (en tenant compte du phénomène de condensation lorsque cela est nécessaire), et que l'on porte $q^{*}$ en fonction de $2 \pi / \xi$ (figure $8 \mathrm{~b}$ ), on voit apparaître une courbe maîtresse. Les valeurs expérimentales de $q^{*}$ peuvent donc être correctement reproduites par l'approche en loi d'échelle du modèle isotrope. Si celle-ci a été initialement développée dans le cadre des PEs faiblement chargés, elle semble finalement vérifiée quel que soit le taux de charge, à condition de remplacer le taux de charge chimique $f$ par le taux de charge effectif $f_{\text {eff }}$. Le modèle isotrope semble décrire de façon satisfaisante la structure des solutions de PEs flexibles.

Notons qu'on ne peut s'affranchir d'une légère dispersion sur la courbe maîtresse. Comme nous l'avons vu, les mesures de $q^{*}$ sont également sensibles aux facteurs de contraste des monomères de différents constituants (cf. 5.2). 


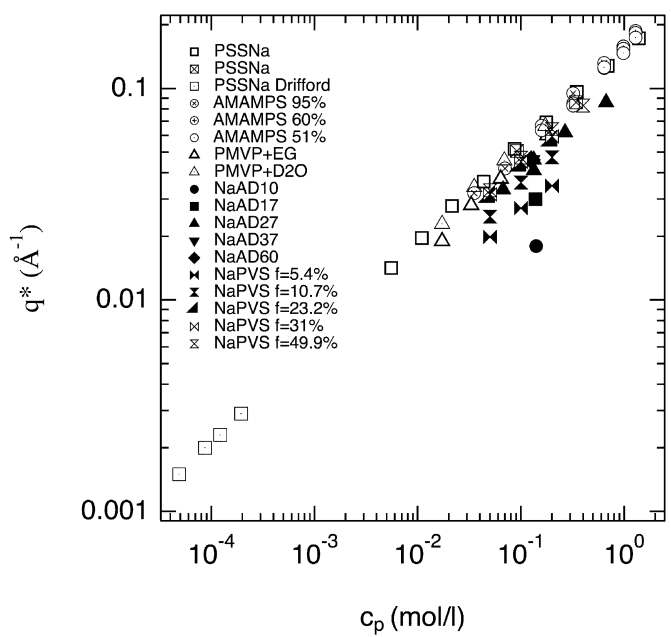

a)

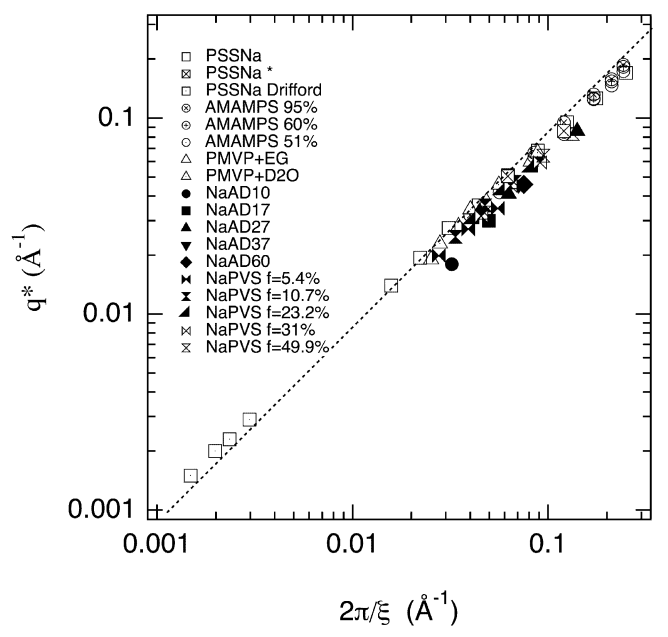

b)

Figure 8. a) Position du maximum $q^{*}$ pour différents systèmes (différents polyions et différents contreions monovalents, pour différents taux de charge $f$, dans différents solvants, et de part et d'autre du seuil de condensation de Manning-Oosawa) en fonction de la concentration $c_{p}$ [15]. b) Position du maximum $q^{*}$ en fonction de $2 \pi / \xi$.

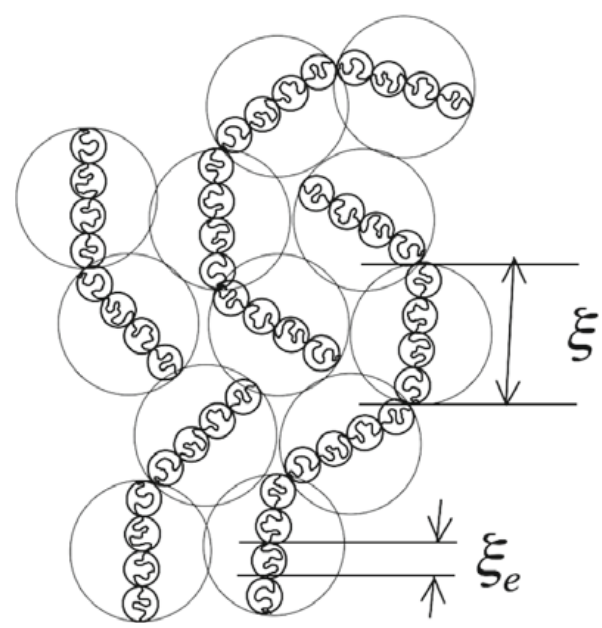

Figure 9. Représentation schématique d'une solution de PEs suivant l'approche de loi d'échelle associée au modèle isotrope. Dans cette représentation bidimensionnelle, les bouts de chaîne représentent en fait des bifurcations hors du plan. La solution semidiluée est composée d'un empilement compact de blobs de corrélation de taille $\xi$.

\section{FONCTIONS DE STRUCTURE INTRA ET INTERMOLÉCULAIRE}

Les exemples présentés jusqu'ici mettent en jeu les fonctions de structure partielles des différents constituants. Il est impossible d'en tirer des renseignements précis et surtout directs, sur la conformation moyenne des diffuseurs ainsi que sur les véritables corrélations intermoléculaires. Lorsque ces informations sont nécessaires, il faut essayer de séparer les contributions intra et intermoléculaire. Ceci peut être réalisé par diffusion de neutrons en tirant profit de la substitution isotopique H/D comme indiqué dans la partie 4.2. Dans tout ce qui suit, nous considèrerons que la contribution des contreions 
à l'intensité mesurée est négligeable. Une présentation détaillée des facteurs de forme des polymères en solution est donnée en [30].

\subsection{Facteur de forme}

La fonction de structure intramoléculaire (ou facteur de forme) $g_{1}(q)$ permet de caractériser la forme moyenne des polyions. Elle recouvre leur taille caractéristique (rayon de giration $R_{g}$ ), le degré de polymérisation $N$, et la conformation moyenne des polyions. On distingue généralement deux régimes suivant que l'on sonde des distances plus grandes, ou plus petites que le rayon de giration des chaînes. Cela correspond au domaine de Guinier $\left(q R_{g}<1\right)$ et au domaine asymptotique $\left(q R_{g}>1\right)$.

\subsubsection{Domaine de Guinier}

Dans le domaine de Guinier $\left(q \cdot R_{g}<1\right)$, la résolution spatiale permet de sonder des distances à l'échelle macromoléculaire. On peut alors déterminer l'indice de polymérisation et le rayon de giration des polyions. Ce dernier s'exprime d'un point de vue mathématique comme :

$$
R_{g}^{2}=\frac{1}{2 N^{2}} \sum_{i, j}\left\langle\left(\vec{r}_{i}-\vec{r}_{j}\right)^{2}\right\rangle .
$$

Dans cette expression, la sommation est à effectuer sur toutes les positions $\vec{r}_{i}$ et $\vec{r}_{j}$ des monomères $i$ et $j$ le long d'un polyion, et $<>$ représente une moyenne d'ensemble. Les polymères étant des particules statistiques, ils fluctuent dans le temps et dans l'espace. Leur rayon de giration représente une valeur moyenne sur l'ensemble des états accessibles à l'échelle de $k_{B} T$. Rappelons également que le formalisme développé dans cette partie ne s'applique qu'aux homopolymères, c'est-à-dire aux polymères constitués d'un seul type de monomère. Dans le cas de copolymères l'analyse est légèrement plus complexe et peut conduire à des rayons de giration apparents positifs, nuls voire négatifs [30].

Le facteur de forme d'une particule est une fonction qui décroît généralement de façon monotone et tend vers zéro pour les plus grands vecteurs de diffusion $q$. Dans le domaine de Guinier $\left(q R_{g}<1\right)$, il peut s'approximer par un développement limité qui prend la forme :

$$
g_{1}(q)=N\left[1-\frac{q^{2} R_{g}^{2}}{3}+\circ\left(q^{2}\right)\right] .
$$

Pour des macromolécules linéaires, on préfère généralement utiliser les représentations de Zimm [31] ou de Berry [32] :

$$
\begin{aligned}
& \frac{1}{g_{1}(q)}=\frac{1}{N}\left[1+\frac{q^{2} R_{g}^{2}}{3}+\circ\left(q^{2}\right)\right] \text { Zimm } \\
& \frac{1}{\sqrt{g_{1}(q)}}=\frac{1}{\sqrt{N}}\left[1+\frac{q^{2} R_{g}^{2}}{6}+\circ\left(q^{2}\right)\right] \text { Berry. }
\end{aligned}
$$

Un tracé de $1 / g_{1}(q)$, ou de $1 / \sqrt{g_{1}(q)}$ en fonction de $q^{2}$ nous permet de mesurer le rayon de giration des chaînes (pente de la droite), et l'extrapolation de $g_{1}(q)$ à $q=0$, de déterminer le degré de polymérisation $N$. Notons que ce dernier réclame que l'on ait pris soin de travailler a une échelle absolue d'intensité. Connaissant la masse molaire des monomères $m$, on peut déduire la masse moléculaire du polyion $M=N m$.

La détermination expérimentale du rayon de giration et du degré de polymérisation n'est pas toujours simple à réaliser. Pour des chaînes relativement longues, le régime de Guinier peut se limiter aux très faibles vecteurs de diffusion. Dans certains cas, il peut même sortir de la gamme accessible au 
diffractomètre. Une chaîne de rayon de giration de $100 \AA$ conduit à une limite du régime de Guinier $q_{\text {limite Guinier }}=0.01 \AA^{-1}$. Pour déterminer $R_{g}$, il faudrait commencer les mesures vers $0.003 \AA^{-1}$, ce qui est loin d'être toujours évident. De plus, cette gamme de vecteurs de diffusion peut présenter des remontées parasites (cf. 5.1), indépendantes des propriétés macromoléculaires individuelles, qui peuvent compliquer l'analyse et conduire à des valeurs de $R_{g}$ et de $N$ erronées. L'étude du facteur de forme aux faibles vecteurs de diffusion doit donc s'effectuer avec le plus grand soin.

Lorsque l'on est confronté à des problèmes de polymolécularité, on mesure la moyenne dite en masse $(w)$ du facteur de forme $\left\langle g_{1}(q)\right\rangle_{w}$ [30]. La représentation de Zimm prend alors la forme :

$$
\frac{1}{\left\langle g_{1}(q)\right\rangle_{w}}=\frac{1}{N_{w}}\left[1+\frac{q^{2}\left\langle R_{g}^{2}\right\rangle_{z}}{3}+\circ\left(q^{2}\right)\right]
$$

dans laquelle $N_{w}$ est la moyenne $w$ du degré de polymérisation $N,\left\langle R_{g}^{2}\right\rangle_{z}$ est la moyenne z du carré du rayon de giration.

\subsubsection{Domaine asymptotique}

Dans le domaine asymptotique $\left(q R_{g}>1\right)$, on sonde la structure interne des polyions. Les résultats ne sont plus sensibles à la polymolécularité. L'analyse des données se fait généralement sur la base d'une comparaison à des modèles. Des mesures réalisées sur le polystyrène sulfonate (PSSNa) [33] ont démontré que le rayon de giration des chaînes était compris entre celui d'une chaîne gaussienne et celui d'un bâtonnet rigide, traduisant un étirement global des macromolécules sous l'effet des répulsions électrostatiques. Le modèle adopté pour décrire ce type de conformation moyenne est celui de la chaîne à longueur de persistance ( «wormlike chain », ou encore chaîne de Kratky-Porod).

La chaîne (supposée monodisperse et sans épaisseur) adopte une statistique gaussienne à faible résolution (grande distance, faibles valeurs de $q$ ) qui évolue vers une conformation en bâtonnet pour des distances inférieures à la longueur de persistance $l_{p}$ (grands $q$ ). Le facteur de forme ne dépend que de la longueur de contour de la chaîne étirée $L(L=N b)$ et de la longueur de persistance $l_{p}$. Il n'existe pas d'expression analytique simple valable sur tout le domaine de $q$. Le facteur de forme global $g_{1}(q)$ peut toutefois être obtenu en juxtaposant 3 fonctions $f_{1}(q), f_{2}(\mathrm{q})$ et $f_{3}(q)$ suivant le domaine de $q . l_{p}$ considéré [6, 30, 34-36].

Pour les plus faibles vecteurs de diffusion et lorsque les conditions $L \geq 20 l_{p}$ et $q l_{p} \leq 1.5$ sont réunies (région 1), on peut considérer le facteur de forme déterminé par Sharp et Bloomfield [34] :

$$
\begin{aligned}
g_{1}(q) & =f_{1}(q)=N\left(\frac{2}{x^{2}}[x-1+\exp (-x)]+\frac{2}{l_{P}}\left[\frac{4}{15}+\frac{7}{15 x}-\left(\frac{11}{15}+\frac{7}{15 x}\right)\right] \exp (-x)\right) \\
x & =N q^{2} b l_{P} / 3
\end{aligned}
$$

Pour $1.5 \leq q l_{p} \leq 3.5$ (région 2), on peut utiliser le développement en fractions continues de des Cloiseaux qui est valable pour une chaîne infinie, et que l'on peut représenter par [35] :

$$
g_{1}(q)=f_{2}(q)=\frac{1}{q^{2} b l_{p}}\left(5.967-0.0177 q l_{p}+0.5976\left(q l_{p}\right)^{2}-0.037\left(q l_{p}\right)^{3}\right) .
$$

Pour $q . l_{p} \geq 3.5$ (région 3 ), on peut utiliser le comportement asymptotique ${ }^{5}$ calculé par des Cloiseaux [35] :

$$
g_{1}(q)=f_{3}(q)=\frac{\pi}{q b}+\frac{2}{3} \frac{1}{q^{2} b l_{P}}=\frac{\pi M_{L}}{m q}+\frac{2}{3} \frac{M_{L}}{q^{2} m l_{P}}
$$

\footnotetext{
5 D'autres coefficients numériques associés à des domaines de validité $q l_{p}$ légèrement différents peuvent également être rencontrés dans la littérature. Les différences sont généralement négligeables.
} 


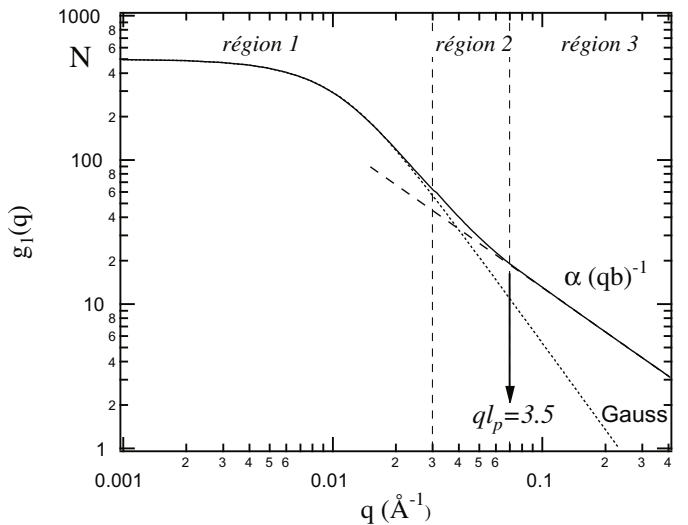

a)

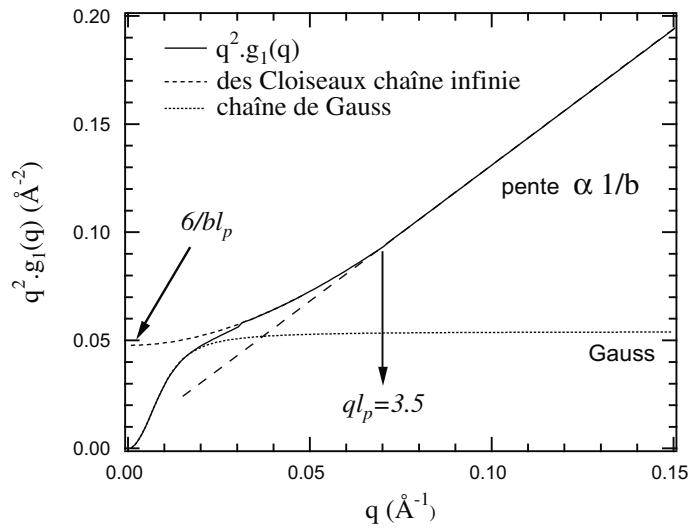

b)

Figure 10. Facteur de forme d'une chaîne à longueur de persistance en représentation logarithmique (a) et en représentation de Kratky (b). $N=500, b=2.5 \AA$ et $l_{p}=50 \AA$ A Les 3 régions associées aux fonctions $f_{1}(q), f_{2}(q)$ et $f_{3}(q)$ sont indiquées par des tirets verticaux. Le facteur de forme d'une chaîne gaussienne de même rayon de giration (136 ̊̊) est donné dans chacune des deux représentations.

dans cette expression, $m$ est la masse d'un monomère et $M_{L}$ la masse par unité de longueur de contour de la chaîne $\left(M_{L}=N m / L\right)$.

Le facteur de forme du modèle de la chaîne à longueur de persistance est présenté sur la figure 10 en représentation logarithmique (a), et en représentation de Kratky $\left(q^{2} g_{1}(q)\right.$ en fonction de $\left.q\right)(\mathrm{b})$.

Aux plus grandes valeurs de $q$ (région 3 ), $g_{1}(q)$ présente une décroissance en $q^{-1}$ caractéristique de la diffusion par des bâtonnets. Dans cette gamme de vecteurs d'ondes, on sonde la structure à une échelle inférieure à la longueur de persistance. On s'attend à ce que les chaînes soient rigides et forment de petits objets cylindriques. En représentation de Kratky, cela se traduit par une variation linéaire dont la pente est directement reliée à l'inverse de la taille du monomère $\left(q^{2} g_{1}(q)=\pi / q b\right)$. Une analyse dans ce domaine de diffusion permet en principe de remonter au seul paramètre $b$,ou encore, à la masse par unité de longueur $M_{L}$.

Lorsque $q l_{p} \leq 3.5, q^{2} g_{1}(q)$ commence à s'écarter du comportement asymptotique et amorce un début de plateau dont la hauteur (déterminée à partir $f_{2}(q)$ dans le cas des chaînes infinies) est donnée par $6 / b l_{p}$. La chaîne étant de longueur finie, le plateau n'est jamais atteint et $q^{2} g_{1}(q)$ décroît à nouveau. La fin du régime linéaire $\left(q l_{p}=3.5\right.$ ) (ou du régime en $q^{-1}$ en représentation logarithmique) peut être mise à profit pour déterminer de façon relativement simple, la longueur de persistance $l_{p}$.

Aux plus petits vecteurs de diffusion, la chaîne se comporte comme une chaîne gaussienne. $g_{1}(q)$ tend vers un plateau et on entre dans le régime de Guinier pour une chaîne de longueur finie. L'extrapolation de $g_{1}(q)$ à $q=0$ nous renseigne alors comme nous l'avons vu précédemment, sur le degré de polymérisation $N$.

Le comportement gaussien aux plus petits vecteurs de diffusion s'étend au-delà du régime de Guinier $\left(q_{\text {limite Guinier }}=7 \cdot 10^{-3} \AA^{-1}\right)$. Le rayon de giration peut donc être déterminé de façon plus confortable en analysant $g_{1}(q)$ sur une gamme de vecteur de diffusion étendue $\left(q R_{g} \leq 3\right)$ à l'aide du facteur de forme d'une chaîne gaussienne (fonction de Debye) [37] :

$$
\begin{aligned}
g_{1}(q) & =N \frac{2}{x^{2}}[x-1+\exp (-x)] \\
x & =q^{2} R_{g}^{2}
\end{aligned}
$$


$R_{g}^{2}$ s'exprime en fonction des paramètres $N, b$ et $l_{p}$ (relation de Benoît Doty [38]) :

$$
R_{g}^{2}=\frac{N b l_{P}}{3}\left[1-\frac{3 l_{P}}{N b}\left(1-\frac{2 l_{P}}{N b}\right)-\frac{6 l_{P}^{3}}{(N b)^{3}}\left(1-\exp \left(-\frac{N b}{l_{P}}\right)\right)\right] .
$$

Il est donc possible à partir de $R_{g}$ de remonter à la longueur de persistance de la chaîne (si l'on connaît la longueur totale du polyion $\mathrm{Nb}$ ) vice-versa si les valeurs de $l_{p}$ et de $N b$ sont connues. Une analyse précise nécessite néanmoins de prendre en compte la polymolécularité des macromolécules sans quoi des erreurs importantes peuvent apparaître [30,33].

D'un point de vue expérimental, la mesure du facteur de forme n'est pas toujours simple à réaliser [36, 39-41]. Les expériences dans les conditions de contraste moyen nul ne diffusent généralement pas beaucoup. Des problèmes de statistique peuvent en particulier apparaître aux plus grands vecteurs de diffusion. La soustraction du bruit de fond peut alors affecter la pente de $q^{2} g_{1}(q)$ et introduire des erreurs sur $b$. D'autre part, à partir d'une certaine échelle spatiale, les monomères ne peuvent plus être considérés comme ponctuels. L'affinement des données doit alors prendre en compte un terme de section supplémentaire. Si l'on considère les chaînes comme des cylindres de rayon de giration axial $R c$, le facteur de forme doit alors être remplacé par :

$$
g_{1}(q) \exp \left(-\frac{q^{2} R c^{2}}{2}\right)
$$

pour des valeurs de $q R c<1$ (régime de Guinier du terme de section).

Un exemple de facteur de forme expérimental est présenté sur la figure 11. Il a été mesuré sur une solution de PSSNa $\left(f=1, c_{p}=0.17 \mathrm{~mol} / \mathrm{L}\right)$ par la méthode du contraste moyen nul. Les échantillons étaient composés de mélanges 50/50 de chaînes $\mathrm{H}$ et $\mathrm{D}$ de même de gré de polymérisation, dans un solvant $29 \% \mathrm{H}_{2} \mathrm{O} / 71 \% \mathrm{D}_{2} \mathrm{O}$. Dans ces solutions, la contribution des contreions sodium à l'intensité diffusée est négligeable [41].

L'extrapolation de $g_{1}(q)$ à $q=0$ nous permet de déterminer la moyenne en poids de l'indice de polymérisation $N_{w}=730$, valeur assez proche de celle déterminée par chromatographie d'exclusion stérique $\left(N_{w}=700\right)$. L'influence du terme de section commence à apparaître à des valeurs de $q$ relativement faibles limitant le domaine d'observation du régime en $q^{-1}$ sur $g_{1}(q)$, ou du régime linéaire en représentation de Kratky.

\subsection{Fonction de structure intermoléculaire}

La fonction de corrélation de paires $g\left(q, c_{p}\right)$ (ou de la fonction de structure totale $S_{T}\left(q, c_{p}\right)$ ) et le facteur de forme $g_{1}\left(q, c_{p}\right)$ (ou de la fonction de structure intrachaîne $S_{1}\left(q, c_{p}\right)$ ) permettent de remonter à la fonction de corrélation intermoléculaire $g_{2}\left(q, c_{p}\right)$ (ou à la fonction de structure intermoléculaire $S_{2}\left(q, c_{p}\right)$ ). Il est assez courant de rencontrer dans la littérature des PEs, un facteur de structure apparent $S_{G}(q, c)$ défini comme

$$
S_{G}\left(q, c_{p}\right)=S_{T}\left(q, c_{p}\right) / S_{1}\left(q, c_{p}\right)=1+c_{p} \frac{g_{2}\left(q, c_{p}\right)}{g_{1}\left(q, c_{p}\right)} .
$$

Cette relation n'est théoriquement valable que pour des objets à symétrie sphérique. Pour des PEs linéaires en régime semidilué, $S_{G}\left(q, c_{p}\right)$ ne représente plus un véritable facteur de structure, son utilisation doit être réalisée avec précaution. Elle permet néanmoins d'observer facilement des variations sur les corrélations intermoléculaires.

Un exemple de séparation intra/inter est présenté sur la figure 12. Les mesures ont été effectuées sur des échantillons de PSSNa (a) et $\operatorname{PSSCa}_{1 / 2}(\mathrm{~b})$, totalement chargés $(f=1)$ et à $c_{p}=0.17 \mathrm{~mol} / \mathrm{L}$. La fonction de structure intramoléculaire $S_{1}(q)$ a été mesurée dans les mêmes conditions que décrites précédemment. La fonction de structure totale $S(q)$ a été mesurée avec des chaînes hydrogénées 


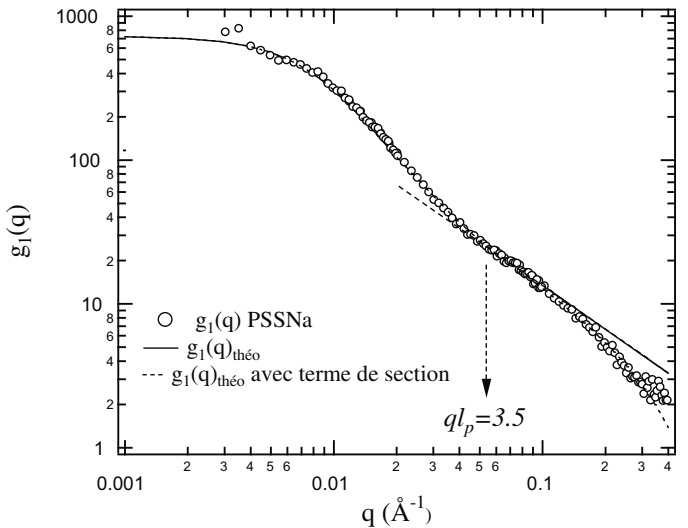

a)

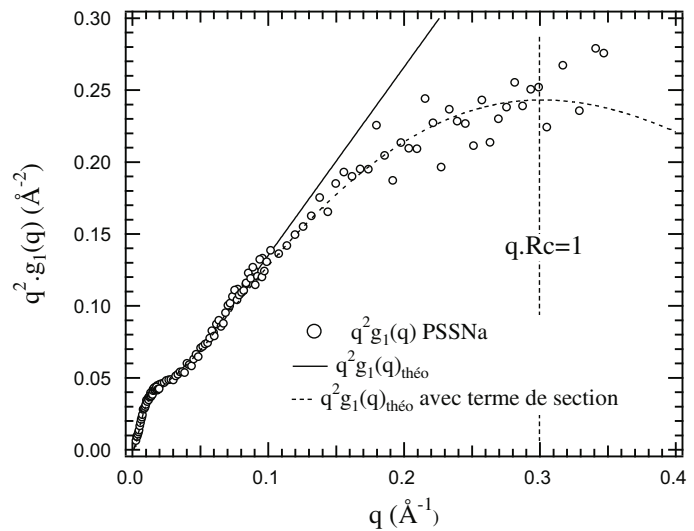

b)

Figure 11. Facteur de forme expérimental d'une chaine de PSSNa, $c_{p}=0.17 \mathrm{~mol} / \mathrm{L}, f=1$ (D22, ILL, Grenoble). Comparaison avec le modèle de la chaîne à longueur de persistance avec et sans terme de section. $N=730, b=2.4$ $\AA$ et $l_{p}=65 \AA, R c=3.3 \AA$. Représentation logarithmique (a) et représentation de Kratky (b).

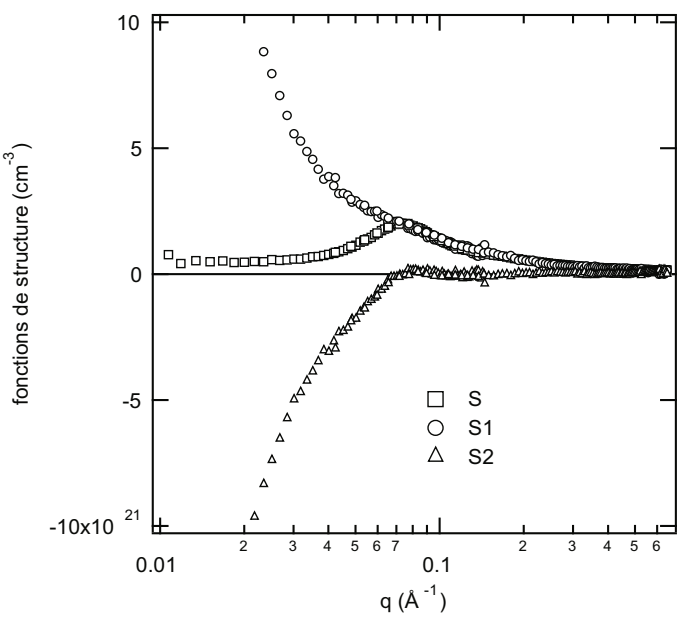

a)

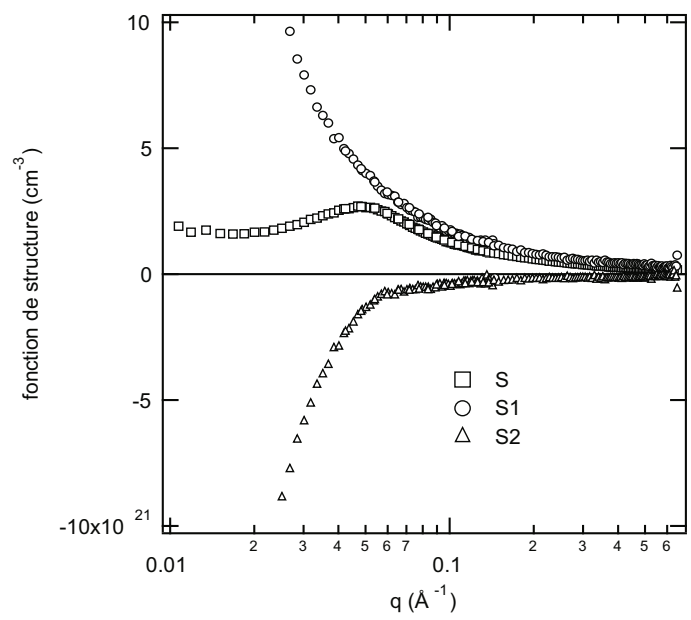

b)

Figure 12. Fonctions de structure totale $(S)$, intramoléculaire $\left(S_{1}\right)$ et intermoléculaire $\left(S_{2}\right)$ pour des échantillons de PSSNa (a) et PSSCa $\operatorname{Pa}_{1 / 2}$ (b) à $c_{p}=0.17 \mathrm{~mol} / \mathrm{L}$. Les mesures ont été réalisées sur D22 à l'ILL. $S_{1}$ a été mesurée dans les conditions de contraste moyen nul. $S$ a été mesurée avec des chaînes $\mathrm{H}$ dans $\mathrm{D}_{2} \mathrm{O}$.

dans $\mathrm{D}_{2} \mathrm{O}$. La fonction de structure intermoléculaire est alors déterminée par la relation $S_{2}\left(q, c_{p}\right)=$ $S\left(q, c_{p}\right)-S_{1}\left(q, c_{p}\right)$.

L'interprétation des fonctions de structure intermoléculaire n'est pas simple à réaliser principalement en raison de la symétrie anisotrope des macromolécules. Différentes propriétés peuvent toutefois être clairement mises en évidence.

La fonction de structure intermoléculaire $S_{2}(q)$ est négative pour les plus faibles valeurs de $q$, traduisant le caractère répulsif des interactions électrostatiques. Elle tend vers zéro pour les plus grands vecteurs de diffusion. Au-delà d'une certaine valeur de $q$, la fonction de structure totale $S(q)$ devient donc égale à la fonction de structure intramoléculaire $S_{1}(q)$. Ceci nous amène à quelques commentaires sur les fonctions de diffusion partielles présentées dans la partie 5. Lorsque l'on mesure uniquement 
$S_{m m}$, l'intensité mesurée doit reproduire le facteur de forme des chaînes pour peu que l'on s'éloigne franchement de la position du pic de diffusion. Cette remarque avait déjà été évoquée dans le paragraphe 5.1. Le facteur de forme variant théoriquement en $q^{-1}$ aux plus grands vecteurs de diffusion, il devrait en être de même pour $S_{m m}$. En réalité, ce domaine étant souvent réduit à cause du terme de section, les variations expérimentales rencontrées sont souvent plus rapides. Lorsque les mesures sont réalisées sans condition de contraste particulière (PSSNa, rayons X), toutes les fonctions de structure partielles interviennent. La décroissance en en $q^{-1}$ n'est jamais observée.

Dans le cas de PSSNa, $S_{2}(q)$ présente un petit maximum positif (suivi d'une large oscillation) à une position très légèrement supérieure à celle rencontrée dans $S(q)$. Il traduit vraisemblablement l'arrangement régulier d'un petit nombre de chaînes (ou de certaines portions de celles-ci) dans la solution. Sa contribution au pic PE observé dans la fonction de diffusion totale $S(q)$ reste néanmoins assez faible. La fonction de structure intermoléculaire $S_{2}(q)$ de $\operatorname{PSSCa}_{1 / 2}$ ne présente aucun maximum similaire.

Pour ces deux systèmes, le pic PE résulte donc essentiellement (PSSNa), voire complètement $\left(\operatorname{PSSCa}_{1 / 2}\right)$, de la seule combinaison d'une fonction de structure décroissante $\left(S_{1}(q)\right)$ et d'une fonction de structure croissante $\left(S_{2}(q)\right)$. Toutes ces observations sont compatibles avec un réseau isotrope désordonné («trou de corrélation») comme décrit dans le paragraphe 5.4. Les solutions peuvent dans certains cas comporter une petite fraction de chaînes (ou de partie de chaînes) ordonnées (PSSNa). La différence entre PSSNa et $\mathrm{PSSCa}_{1 / 2}$ pourrait alors être reliée à la présence des pontages électrostatiques qui déstabilisent l'organisation et empêchent l'alignement des chaînes (cf. 5.3).

Notons enfin que si la position du pic de diffusion observé sur la fonction de structure totale $S(q)$ de PSSNa et la position du maximum de la fonction de structure intermoléculaire associée $S_{2}(q)$ sont très proches l'une de l'autre, cela n'est plus le cas lorsque l'on rajoute du sel et que l'on écrante les interactions électrostatiques [42].

\section{CONCLUSION}

Dans cette contribution, nous avons présenté l'apport de la diffusion de rayons $\mathrm{X}$ et des neutrons à l'étude des solutions de PEs. Nous avons essayé de mettre en évidence les principales caractéristiques des courbes de diffusion et montré les informations que l'on pouvait en tirer au regard de théories, ou de modèles relativement simples. Nous nous sommes toutefois limités aux PEs flexibles, hydrophiles, en régime semidilué et sans sel ajouté.

Les mesures réalisées sans condition de contraste particulière permettent de déterminer la section efficace différentielle cohérente par unité de volume $\Sigma^{c o h}(q)$ qui contient toute l'information sur la structure du soluté. Celle-ci peut s'exprimer comme une combinaison linéaire des fonctions de structure partielles $S_{m m}, S_{c c}$ et $S_{m c}$ pondérées par les longueurs de contraste des différents constituants. La diffusion de neutrons, grâce à la substitution isotopique, offre un avantage important, puisqu'elle permet d'annuler ou au contraire de révéler la contribution des macroions ou des contreions.

La section efficace cohérente $\Sigma^{c o h}(q)$ présente un maximum qui se déplace avec la concentration et le taux de charge. L'étude de sa position permet de sonder principalement l'état de dispersion des chaînes dans la solution (et indirectement, via l'approche en loi d'échelle, la conformation moyenne des macromolécules). La comparaison avec des modèles théoriques semble indiquer la formation d'un réseau isotrope désordonné. Dans cette approche, les chaînes se repoussent et ne peuvent se rapprocher en deçà d'une distance minimale («trou de corrélation électrostatique »). C'est cette longueur caractéristique que l'on sonde en mesurant $q^{*}$. Si un ordre orientationnel ou translationnel dans lequel les chaînes (ou des parties de celles-ci) s'arrangent de façon plus régulière ne peut-être exclu, il n'est cependant pas majoritaire dans la solution.

L'évolution caractéristique en $c_{p}^{1 / 2}$ sur une gamme de concentration raisonnable est caractéristique d'un système de chaînes linéaires où les interactions sont principalement de nature électrostatique. 
Lorsque les chaînes présentent un caractère hydrophobe important (non présenté dans cette contribution), l'évolution de $q^{*}$ se trouve modifiée et différents régimes apparaissent.

L'étude du maximum de diffusion présente encore d'autres intérêts. Sa sensibilité à la fraction de charge nette, en fait une sonde pour l'étude du phénomène de condensation des contreions. Cette condensation peut également être mise en évidence en mesurant la fonction de structure partielle associée aux contreions. La présence d'un pic dans $S_{c c}$ à une position similaire de celle rencontrée dans $S_{m m}$ est une conséquence directe de l'accumulation des contreions dans le proche voisinage des chaînes. D'autre part, lorsque l'on s'éloigne du pic PE et que l'on sonde les plus grands vecteurs de diffusion, on rencontre un maximum assez large (ou, une remontée si la gamme de $q$ n'est pas assez importante) qui correspond à la première oscillation du facteur de forme des contreions condensés.

La diffusion de neutrons, grâce au marquage isotopique, permet également d'extraire le facteur de forme des macroions, ainsi que la fonction de structure intermoléculaire.

La conformation moyenne des macroions fortement chargés en solution peut être décrite par le modèle de chaîne à longueur de persistance. Elle se comporte comme une chaîne gaussienne aux plus petits vecteurs de diffusion et évolue vers une conformation en bâtonnet lorsque l'on sonde des distances plus petites que la longueur de persistance. Les mesures réalisées dans le régime de Guinier déterminent un rayon de giration, ainsi qu'un degré de polymérisation. Dans le régime asymptotique, elles permettent de déterminer la taille des monomères, ainsi que la longueur de persistance. Il faut toutefois prendre en compte un terme supplémentaire lié à la section de la chaîne pour analyser parfaitement les données.

La décomposition de l'intensité diffusée en contributions intra et intermoléculaire permet de comprendre l'origine du pic de diffusion observée dans $\Sigma^{c o h}(q)$. Si, dans certains cas, le facteur de structure intermoléculaire montre un léger maximum, caractéristique d'une organisation plus régulière de certaines portions de chaînes, celui-ci ne contribue que très peu à l'intensité du pic PE. Celui-ci résulte essentiellement de la combinaison d'une fonction croissante (facteur de structure intermoléculaire) et décroissante (facteur de structure intramoléculaire).

\section{Remerciements}

Ce cours repose essentiellement sur les recherches que j'effectue à l'Institut Charles Sadron avec Michel Rawiso sur les solutions de polyélectrolytes. Je tiens à le remercier tout particulièrement pour sa relecture critique ainsi que pour les nombreuses discussions fructueuses que nous avons eues à ce sujet. Je tiens également à remercier mes collègues qui ont participé à la relecture de l'article, ou aux différentes expériences présentées : François Boué (LLB, Saclay), Isabelle Grillo (ILL, Grenoble), Fabrice Cousin (LLB, Saclay), Bruno Démé (ILL, Grenoble) et Cyrille Rochas (ESRF / CERMAV, Grenoble).

\section{Références}

[1] G.S. Manning, J. Chem. Phys., 1969, 51, 954.

[2] Oosawa, F. Biopolymers, 1968, 6, 134

[3] W. Essafi, F. Lafuma, D. Baigl, C.E. Williams, Europhys. Lett., 2005, 71, 938.

[4] L. Van Hove, Phys. Rev., 95 (1954), 249.

[5] J. S. Higgins, H.C. Benoît, «Polymers and Neutron Scattering », Oxford University Press, New York 1996.

[6] J.P Cotton, J. Phys. IV France 9, 1999, Pr-21 (Ecole Thématique de la Société Française de Neutronique "Diffusion de Neutrons aux Petits Angles").

[7] J.P. Cotton, M. Moan, J. Phys. Lett., 1976, 37, L-75.

[8] Kaji K., Urakawa H., Kanaya T., Kitamaru R., J. Phys. (France), 1988, 49, 993.

[9] M. Nierlich, C.E. Williams, F. Boué, J.P. Cotton, M. Daoud, B. Farnoux, G. Jannink, C. Picot, M. Moan, C. Wolf, M. Rinaudo, P.-G. de Gennes, J. Phys. (France), 1979, 40, 701.

[10] De Gennes P.-G., Pincus P., Velasco R.M, Brochard F., J. Phys. (France), 1976, 37, 1461.

[11] P. Vallat, J.-M. Catala, M. Rawiso, F. Schosseler, Macromolecules, 2007, 40, 3779. 
[12] K. Nishida, K. Kaji, T. Kanaya, J. Chem. Phys., 2001, 114, 8671.

[13] W. Essafi, F. Lafuma, C.E. Williams, dans «Macro-ion Characterization. From Dilute Solutions to Complex Fluids», édité par Schmitz K. S., ACS Symposium Series, 548 (1994) 278.

[14] Dobrynin V., Rubinstein M., Macromolecules, 1999, 32, 915.

[15] J. Combet, F. Isel, M. Rawiso, F. Boué, Macromolecules, 2005, 38, 7456.

[16] B.D. Ermi, E.J. Amis, Macromolecules, 1998, 31, 7378.

[17] J.R.C. van der Maarel, L.C.A. Groot, J.G. Hollander, W. Jess, M.E. Kuil, J.C. Leyte, L.H. LeyteZuiderweg, M. Mandel, J.P. Cotton, G. Jannink, A. Lapp, B. Farago, Macromolecules, 1993, 26, 7295.

[18] L.B. Bhuiyan, C.W. Outhwaite, J.R.C. van der Maarel, Physica A, 1996, 231, 295.

[19] K. Kassapidou, W. Jesse, M.E. Kuil, A. Lapp, S. Egelhaaf, J.R.C. van der Maarel, Macromolecules, 1997, 30, 2671.

[20] Spiteri M.N., Williams C.E., Boué F., Macromolecules 40, 2007, 6679.

[21] D. Baigl, Thèse de doctorat, Université Paris VI "Etude expérimentale de polyélectrolytes hydrophobes modèles", 2003.

[22] K. El Brahmi, Thèse de doctorat, Université de Strasbourg, 1991.

[23] Nishida K., Kaji K., Kanaya T., Macromolecules, 1995, 28, 2472.

[24] Essafi W., Lafuma F., Williams C.E., Eur. Phys. J. B, 1999, 9, 261.

[25] Qu D., Pedersen J.S., Garnier S., Laschewsky A., Möhwald H., v. Klizing R., Macromolecules, 2006, 39, 7364.

[26] J. Combet, M. Rawiso, F. Boué, C. Rochas, Macromolecules, 2010 soumis.

[27] R. Koyama, Macromolecules, 1984, 17, 1594.

[28] P.J. Pfeuty, J. Phys., 1978, 39, C2-149.

[29] Dobrynin A.V., Colby R.H., Rubinstein M., Macromolecules, 1995, 28, 1859.

[30] M. Rawiso, J. Phys. IV France 9, 1999, Pr-147 (Ecole Thématique de la Société Française de Neutronique "Diffusion de Neutrons aux Petits Angles").

[31] B.H. Zimm, J. Chem. Phys., 1948, 16, 1099.

[32] G.C. Berry, J. Chem. Phys., 1966, 44, 4550.

[33] M. Nierlich, F. Boué, A. Lapp, R. Oberthur, J. Physique, 46 (1985) 649-655

[34] P. Sharp, V.A. Bloomfield, Biopolymers, 6, (1968), 1201

[35] J. De Cloiseaux, Macromolecules, 6, (1973), 403.

[36] A. Brûlet, F. Boué, J.P. Cotton, J. Phys. II France, 6, 1996, 885

[37] P. Debye, J. Chem. Phys., 1946, 14, 636.

[38] H. Benoît, P.M. Doty, J. Phys. Chem., 87 (1953) 958.

[39] M. Ragnetti, R.C. Oberthür, Collö̈ds \& Polymer Science, 1986, 264, 32.

[40] M.N. Spiteri, Thèse de doctorat, Université Paris XI "Conformation et arrangement des polyélectrolytes en solution semidiluée. Etude par diffusion des neutrons aux petits angles", 1997.

[41] E. Dubois, F. Boué, Macromolecules, 2001, 34, 3684.

[42] K. Nishida, K. Kaji, T. Kanaya, T. Shibano, Macromolecules, 2002, 35. 